\title{
Interacting and merging galaxies from the second Byurakan survey ${ }^{\star}$ (Research Note)
}

\section{HI-observations}

\author{
W. K. Huchtmeier ${ }^{1}$, A. Petrosian ${ }^{2}$, G. Krishna ${ }^{3}$, B. McLean ${ }^{4}$, and D. Kunth ${ }^{5}$ \\ 1 Max-Planck-Institut für Radioastronomie, Auf dem Hügel 69, 53121 Bonn, Germany \\ e-mail: huchtmeier@mpifr-bonn.mpg.de \\ 2 Byurakan Astrophysical Observatory and Isaac Newton Institute of Chile, Armenian Branch, Byurakan 378433, Armenia \\ e-mail: artptrs@yahoo.com \\ 3 NCRA-TIFR, Pune University Campus, Pune 411 007, India \\ e-mail: krishna@ncra.tifr.res.in \\ 4 Space Telescope Science Institute, 3700 San Martin Drive, Baltimore, MD21218, USA \\ e-mail: mclean@stsci.edu \\ 5 Institut d'Astrophysique, Paris, France \\ e-mail: kunth@iap.fr
}

Received 1 August 2008 / Accepted 16 September 2008

\section{ABSTRACT}

\begin{abstract}
We present new results from $\mathrm{HI}$ observations of 53 previously undetected interacting and merging galaxies from the Second Byurakan Survey (SBS), in which 30 systems have been detected (detection rate 56\%). High-resolution gray-scale optical images and isophotal maps of the HI detected galaxy systems are presented, in addition to comments on these systems. The possibility of confusion arising from known objects within the telescope beam is discussed for each case. For nine unconfused mergers, global HI parameters are determined. These objects are found to be HI-rich and have normal values of (total) mass-to-luminosity ratios. At least 18 objects in the present sample are found to have radio continuum counterparts above the milli-Jansky level near $1 \mathrm{GHz}$. Throughout this paper we use a value of $H_{0}=72 \mathrm{~km} \mathrm{~s}^{-1} \mathrm{Mpc}^{-1}$ for the Hubble constant.
\end{abstract}

Key words. galaxies: interactions - galaxies: formation - galaxies: evolution

\section{Introduction}

The stellar, ionized and neutral gas contents and the history of star formation (SF) are the key parameters determining the evolution of galaxies. Several factors have been claimed to enhance the SF rate in galaxies beyond the typical value for the given morphological type. In particular, a number of studies (e.g. Kennicutt 1998) have invoked gravitational interaction as a possible mechanism for triggering SF. In the past, many studies have been carried out associating galaxy interaction with enhanced star formation (e.g. Kennicutt et al. 1987; Nikolic et al. 2004). The various tracers of SF employed in different studies include optical colors and $\mathrm{H}_{\alpha}$ flux (e.g. Larson \& Tinsley 1978; Kennicutt et al. 1987), near, mid and far-infrared emission (e.g. Cutri \& McAlary 1985; Heckman 1999), distribution of HII regions and morphological studies (e.g. Hodge 1975), radio-continuum output (e.g. Hummel et al. 1987) and supernova events (e.g., Navasardyan et al. 2001). However, a oneto-one correlation between galaxy-galaxy interactions and SF is not evident. Many interacting systems show either only modest, or practically no signs of SF activity. It is often posited that in

* Figures 1 and 2, Tables 1 and 2, and Appendices A and B are only available in electronic form at http://www . aanda.org triggering SF, pre-existing conditions within the interacting systems as well as their dynamics and mass distributions all play some role, but perhaps the most important is the relation between the dynamical time scale of interaction and the SF time scale. It is contended that only close passages can trigger a starburst (e.g. Barton et al. 2000). Detailed investigations of the relation between interaction and SF show that SF takes place predominantly in the central regions of the galaxies (e.g. Kennicut et al. 1987; Petrosian \& Turatto 1995; Bergvall et al. 2003) and global star formation levels in the galaxies do not differ significantly from those in normal isolated galaxies, which is supported also by theoretical considerations and $N$-body simulations (Mihos \& Hernquist 1996). Nonetheless, cases have been reported of an enhanced SF activity spanning the interacting pair of galaxies (e.g. Wang et al. 2004), or even beyond, into the tidal tails hosting nascent dwarfs (e.g. Duc et al. 2000; Neff et al. 2005), or in the outer shells and giant HII complexes (e.g., Lelievre \& Roy 2000).

Fuelling SF in interacting galaxies requires an adequate and sustained supply of neutral and molecular gas. The origin of this gas can be the entire interacting system, or merely the gas bound to one of the galaxies. Interactions can disrupt the axi-symmetry of the galactic gravitational potential, leading to gas flow from the system to one of its components, or from one component 
to another. For several closely interacting/merging galaxy systems, a number of observational studies have revealed enhanced centrally condensed, as well as widely distributed neutral and molecular gas emissions (e.g., Gordon et al. 2001; Casasola et al. 2004; Iono et al. 2005; Cullen et al. 2007; Maybhate et al. 2007). HI-observations of merging and interacting galaxies have also been carried out to probe the formation mechanisms of merging and interacting systems (e.g., Iyer et al. 2004) and these observations provide vital kinematic information. On average, HI $21-\mathrm{cm}$ emission has been detected from only $25-40 \%$ of merging and interacting galaxies observed (e.g. Horellou \& Booth 1997; Emonts et al. 2006). It can be surmized that the present understanding of correlation between HI content, stellar populations and star formation in interacting galaxies is still at a preliminary stage (e.g. Hibbard et al. 2000). This is mainly because for many systems with HI detection, little information exists about their stellar content and SF distribution, and viceversa. To address this shortcoming, we have started an observational project to study the HI-gas content and distribution, stellar population, SF history and nuclear properties of a sample of galaxies in merging and interacting systems. In the present work we focus on a set of 53 merging and interacting galaxies from the Second Byurakan Survey (SBS) (Petrosian et al. 2002), which previously had not been observed at $21 \mathrm{~cm}$. These interacting/merging SBS galaxies have well defined SF and other types of nuclear activity indicators. $B, R$ and $I$ color surface photometry $(B, R, I)$ and $2 \mathrm{D}$-spectroscopy of these objects is currently underway. Those data will be reported in forthcoming papers. Here we present results of $21 \mathrm{~cm} \mathrm{HI-observations}$ of the 53 interacting/merging SBS galaxies. In Fig. $1^{1}$ we have collected grey-scale images and isophotal maps of the $F$-band images of $30 \mathrm{HI}$-detected galaxies of our sample which were extracted from the photographic plates of the Second Palomar Sky Survey (POSS-II). The contour levels are in arbitrary units. The lowest contour was chosen at about the $3 \sigma$ level of the local background. The scale interval was chosen in order to best illustrate both the inner and outer structure of the galaxies. The field size (and thus magnification) was selected individually for each system to clearly illustrate its morphological structure. This sample is presented in Sects. 2 and 3 we describe its HI-observations and present the HI data and profiles. Section 4 provides comments on some individual galaxies. A brief discussion of the results and the main conclusions are presented in Sect. 5.

\section{Sample selection}

The second Byurakan sky survey (Stepanian 1994, and references therein) was carried out using the 1-m Schmidt telescope of the Byurakan observatory (Armenia), which is equipped with three objective prisms $\left(1.5^{\circ}, 3^{\circ}\right.$, and $\left.4^{\circ}\right)$. Using "baked" photographic plates, a limiting photographic magnitude of about 19.5 could be reached. In addition to discovering peculiar objects with strong UV-excess, the improved spectral resolution of the wider prisms permitted identification of galaxies with even moderately strong emission lines, in the cases lacking UV emission excess. The SBS plates covered a region of the sky bounded by $7^{\mathrm{h}} 40^{\mathrm{m}} \leq \alpha \leq 17^{\mathrm{h}} 15^{\mathrm{m}}$ and $49^{\circ} \leq \delta \leq 61^{\circ}$, corresponding to an area of about 1000 sq. deg. Around 3500 peculiar objects were cataloged in this survey, including 1677 new peculiar galaxies (Stepanian 2005). A detailed morphological study of SBS galaxies led to the discovery of 110 SBS galaxies in 107 mergers and

\footnotetext{
1 The optical images of Fig. 1 is only available in the electronic version.
}

58 galaxies in 47 closely interacting systems (Petrosian et al. 2002).

A merger was defined by Petrosian et al. (2002) as: "two or more galaxies in a common envelope, or an object with two or multiple nuclei". For the latter category, the two nuclei should be comparable in brightness and generally located centrally relative to the outer and inner optical isophotes of the system. Multiple nuclei are often connected with structural features like spiral arms, jets, tails, etc. According to Petrosian et al. (2002), the term interacting galaxy is defined as: two or more galaxies separated from each other but apparently connected by tidal features (tails, bridges, loops etc.). Further, one or more galaxy in the system may exhibit a disturbed structure. Usually, an SBS galaxy is one component of the interacting system, but there are also cases where both components of the system are SBS galaxies, or when a single entry in the SBS catalogue refers to the entire interacting system. Occasionally, it is difficult to distinguish between a merger and an interacting system and the two can indeed be classified within the same scheme (e.g. Borne et al. 1999). The initial list of 168 SBS galaxies in mergers and interacting systems forms the source out of which our sample of 53 objects has been drawn by applying the following two criteria: (i) the SBS galaxy must have an optically determined radial velocity lower than about $9000 \mathrm{~km} \mathrm{~s}^{-1}$ and (ii) the object lacks HI observations. A few galaxies with known HI-emission have been included in the source list (and in Table 1) for consistency checks and/or to improve the signal-to-noise ratio.

\section{H l-observations and basic results}

The HI observations were made using the 100-m radiotelescope, which has a half-power beamwidth of $9.3^{\prime}$ at a wavelength of $21 \mathrm{~cm}$. The 8192-channel autocorrelator (AK90) was split into four filter banks of 2048 channels each, using a $10 \mathrm{MHz}$ bandwidth. This yielded a resolution of about $1 \mathrm{~km} \mathrm{~s}^{-1}$ (but it was broadened to $\sim 10 \mathrm{~km} \mathrm{~s}^{-1}$ by applying a Gaussian filter). A typical observing time of 60 min per source yielded an rms noise of $\sim 4 \mathrm{mJy}$ (the system noise was $30 \mathrm{~K}$ ). Most of the observations were repeated in order to improve the signal-to-noise ratio and reliability. An ON-source position was combined with an OFF-source position once every $10 \mathrm{~min}$. This total power mode improved the baseline stability of the spectra. Frequent measurements of well known continuum sources were made in order to control the pointing and calibration of the telescope. Every two to three hours, a well known line source (e.g., dwarf galaxies) was observed as a system check. The toolbox software of the MPIfR was used for the data reduction. The observed spectra were corrected for moderately curved baselines only; this should not introduce additional errors in the estimated velocities and flux densities of the lines since the line profiles are fairly narrow in all cases. As can be seen from Fig. $2^{2}$ significantly stronger radio frequency interference (RFI) has been encountered than in recent years (e.g. Huchtmeier et al. 2005). As the RFI is variable, repeated observations of the same galaxy system helped in distinguishing the galaxy emission from the RFI. It is obvious from Fig. 2 that the RFI is concentrated in certain frequency ranges, inhibiting detection of faint $\mathrm{HI}-\mathrm{emission}$ in these spectral chanels, while still permiting relatively clean observations in the remaining frequency ranges. For seven of the HI profiles in Fig. 2 we have edited out the strong RFI and replaced the corresponding values with zero.

\footnotetext{
${ }^{2}$ Figure 2 is only available in the electronic version.
} 
Out of the 53 observed galaxies, we detected 30 in HI, a detection rate of $\sim 56 \%$ which is comparable to that found by us (Huchtmeier et al. 2005) for blue compact dwarf galaxies.

Optical (grey scale) images and isophotal maps of the 30 HI-detected galaxies are presented in Fig. 1.

The HI profiles in Fig. 2 are contaminated to varying degrees by any unrelated galaxies falling within the $9.3^{\prime}$ antenna beam. Some of the profiles resemble those of dwarf galaxies (e.g., SBS0806+579A, SBS0943+562A), others are typical of spiral disk systems (e.g., SBS1052+581, SBS1124+599), while some others have complex profiles (e.g. SBS1252+591). Clearly, by themselves, the global HI profiles are of limited value in revealing the details of the interaction kinematics and high spatial resolution imaging is needed for this.

Table $1^{3}$ summarizes the observational data: the galaxy's name in Col. 1; its coordinates (J2000) in Col. 2 (as used for the observations, more precise optical coordinates will be published by Petrosian et al. 2008); the optical dimensions in arcsec corresponding to the blue surface brightness level at $25 \mathrm{mag} \mathrm{arcsec}^{-2}$ (Petrosian et al. 2008) in Col. 3; the blue apparent magnitude (Stepanian 2005; Petrosian et al. 2008) in Col. 4; the optical heliocenctric radial velocity (Stepanian 2005; Petrosian et al. 2008) in Col. 5. The HI data follow, viz., the measured HI flux (Col. 6), the observed peak HI line flux and its rms error in Col. 7 (for non detections only the rms noise is shown), the heliocentric radial velocity derived from the mid-point of the line at $50 \%$ of the peak and its error (Col. 8), and the line-width measured at a level of $50 \%$ of the line peak (Col. 9). Column 10 gives the integrated radio continuum flux at $1.4 \mathrm{GHz}$, taken from the $\mathrm{NVSS}^{4}$.

The derived global parameters for the objects for which we found no evidence for substantial confusion of the HI profile are presented in Table $2^{5}$. Comments on individual galaxies can be found in Appendix A.

\section{Discussion}

We have used the 100-m Effelsberg radio telescope to search for $\mathrm{HI}$ emission in a sample of 53 interacting/merging galaxy systems. In all, 30 of these systems have been detected (detection rate $\sim 56 \%$ ). A thorough investigation of possible confusing objects within the 9.3 arcmin antenna beam yields 21 interacting galaxy systems whose HI profiles are likely to be free of confusion. Likewise, 9 of the galaxy merger systems were found without any obvious confusion. For these 9 galaxies we have derived global parameters, the mass-to-luminosity ratios $M_{\mathrm{HI}} / L_{B}$ and $M_{t} / L_{B}$ as well as the mass-ratio $M_{\mathrm{HI}} / M_{t}$, for comparison with normal disk galaxies. Also, we detected two galaxies having HI masses less than $10^{9} M_{\odot}$ within a distance of $\sim 40 \mathrm{Mpc}$. For most other detected galaxies, the HI mass is found to be in the range $10^{9}$ to $10^{10} M_{\odot}$. The distance-independent $M_{\mathrm{HI}} / L_{B}$ ratio is found to have rather high values, characterising the observed objects as fairly HI-rich. Since such values are generally associated only with HI-rich late-type galaxies, it appears that a number of HI-rich late-type systems have merged within these observed galaxies. The high $M_{\mathrm{HI}} / M_{t}$ values too, underline the HI-richness of these galaxies. The observed $M_{t} / L_{B}$ ratios of 1 to 4 in solar units are typical for normal spiral galaxies. The higher observed values (6 to 9) may have resulted from the rather uncertain incli-

\footnotetext{
3 Table 1 is only available in the electronic version.

${ }^{4}$ NRAO VLA Sky Survey at $20 \mathrm{~cm}$, Condon et al. (1998).

5 The description of Table 2 and the table are only available in the electronic version.
}

nations used in correcting the line widths, leading to rather high values for the total mass estimates for these galaxies.

The distribution of the observed radio fluxes is generally rather shallow, except one radio counterpart which is bright (149 mJy at $1.4 \mathrm{GHz}$, Table 1). Another similarly bright potential radio counterpart is slightly offset from the optical positions and needs confirmation.

Acknowledgements. Based on observations with the 100-m radiotelescope of the MPIfR (Max-Planck-Institut für Radioastronomie) at Effelsberg. We have made extensive use of the NASA/IPAC Extragalactic Database (NED), which is operated by the Jet Propulsion Laboratory, Caltech, under contract with the National Aeronautics and Space Administration, and the Digitized Sky Survey (DSS-2) produced by the Space Telescope Science Institute under US Government grant NAG W-2166.

\section{References}

Barton, E. J., Geller, M. J., \& Kensyon, S. J. 2000, ApJ, 530, 660 Becker, R. H., White, R. L., \& Delfand, D. J. 1995, ApJ, 450, 559 (FIRST) Bergvall, N., Laurikainen, E., \& Alto, S. 2003, A\&A, 405, 31

Borne, K. D., Bushouse, H., Colina, L., et al. 1999, Ap\&SS, 266, 137 Bottinelli, L., Gouguenheim, L., Theureau, G., Voudreau, N., \& Paturel, G. 1999, A\&AS, 135, 429

Casasola, V., Bettoni, D., \& Galletta, G. 2004, A\&A, 422, 941

Catri, R. M., \& McAlary, C. W. 1985, ApJ, 296, 90

Condon, J. J., Cotton, W. D., Greisen, E. W., et al. 1998, AJ, 115, 1693 (NVSS)

Cullen, H., Alexander, P., Green, D. A., et al. 2007, MNRAS, 374, 1185

Duc, P.-A., Brinks, E., Springel, V., et al. 2000, AJ, 120, 1238

Emonts, B. H. C., Morganti, R., Oosterloo, T. A., et al. 2006, AN, 327, 139

Giovanelli, R., Haynes, M. P., Salzer, J. J., et al. 1994, AJ, 107, 2036

Gordon, S., Koribalski, B., \& Jones, K. 2001, MNRAS, 326, 578

Heckman, T. 1999, Ap\&SS, 266, 3

Hibbard, J. E., Vacca, W. D., \& Yun, M. S. 2000, AJ, 119, 1130

Hodge, P. W. 1975, AJ, 202, 619

Hollerou, C., \& Booth, R. 1997, A\&AS, 126, 3

Huchtmeier, W. K., Krishna, G., \& Petrosian, A. 2005, A\&A, 434, 887 (Paper I)

Hummel, E., van der Hulst, J. M., Keel, W. C., \& Kennicutt, R. C., Jr. 1987, A\&AS, 70, 517

Iono, D., Yun, M. S., \& Ho, P. M. P. 2005, ApJS, 158, 1

Iyer, M. G., Simpson, C. E., Gottesman, S. T., \& Malphrus, B. K. 2004, AJ, 128, 985

Karachentsev, I. D., Makarov, D. I., \& Huchtmeier, W. K. 1999, A\&AS, 139, 97

Karachentsev, I. D., Karachentseva, V. E., Huchtmeier, W. K., \& Makarov, D. I. 2004, AJ, 127, 2031 [KKHM]

Kennicutt, R. C., Jr. 1998, ARA\&A, 36, 189

Kennicutt, R. C., Roettinger, K. A., Keel, W. C., et al. 1987, AJ, 93, 1011

Larson, R. B., \& Tinsley, B. M. 1978, ApJ, 219, 46

Lelievre, M., \& Roy, J. 2000, AJ, 120, 1306

Maybhate, A., Msiero, J., Hibbard, J. E., et al. 2007, MNRAS, 381, 59

Mihos, J. C., \& Hernquist, L. 1996, ApJ, 464, 641

Navasardyan, H., Petrosian, A. R., Turatto, M., et al. 2001, MNRAS, 328, 1181

Neff, S. G., Thielker, D. A., Seibert, M., et al. 2005, ApJ, 619, 91

Nikolic, B., Cullen, H., \& Alexander, P. 2004, MNRAS, 355, 874

Petrosian, A. R., \& Turatto, M. 1995, A\&A, 297, 49

Petrosian, A., McLean, B., Allen, R. J., et al. 2002, AJ, 123, 2280

Petrosian, A. R., McLean, B., Allen, R. J., \& MacKenty, J. 2007, ApJS, 170, 33

Petrosian, A. R., McLean, B., Stepanian, J. A., et al. 2008, in preparation

Pustilnik, S. A., Kniazev, A. Y., Lipovetsky, V. A., \& Ugryumov, A. V. 2001, A\&A, 373, 429

Richter, O.-G., \& Huchtmeier, W. K. 1991, A\&AS, 87, 425

Schlegel, D. J., Finkbeiner, D. P., \& Davies, M. 1998, ApJ, 500, 525

Stepanian, J. A. 1994, Dr. Sci. Thesis, Spec. Astrophys. Obs. Nizhnij Arkhyz

Stepanian, J. 2005, RMxAA, 41, 155

Stepanian, J. A., Chavushian, V. H., Carrasco, L., et al. 2002, AJ, 124, 1283

Theureau, G., Bottinelli, L., Coudreau-Durand, N., et al. 1998, A\&AS, 130, 333

Thuan, T. X., \& Martin, G. E. 1981, ApJ, 247, 823

Thuan, T. X., Lipovetsky, V. A., Martin, J.-M., \& Pustilnik, S. A. 1999, A\&A,

139,1

Tully, R. B., \& Fisher, R. J. 1977, A\&A, 54, 661

Tully, R. B., Pierce, J., Huang, J. S., et al. 1998, AJ, 115, 2264

van den Bergh, S., Li, W., \& Filippenko, A. V. 2005, PASP, 117, 773

Verheijen, M. A. W. 2001, ApJ, 563, 694

Wang, Z., Fazio, G. G., Ashby, M. L. N., et al. 2004, ApJS, 154, 193 
W. K. Huchtmeier et al.: Interacting and merging galaxies. I. (RN), Online Material $p 1$
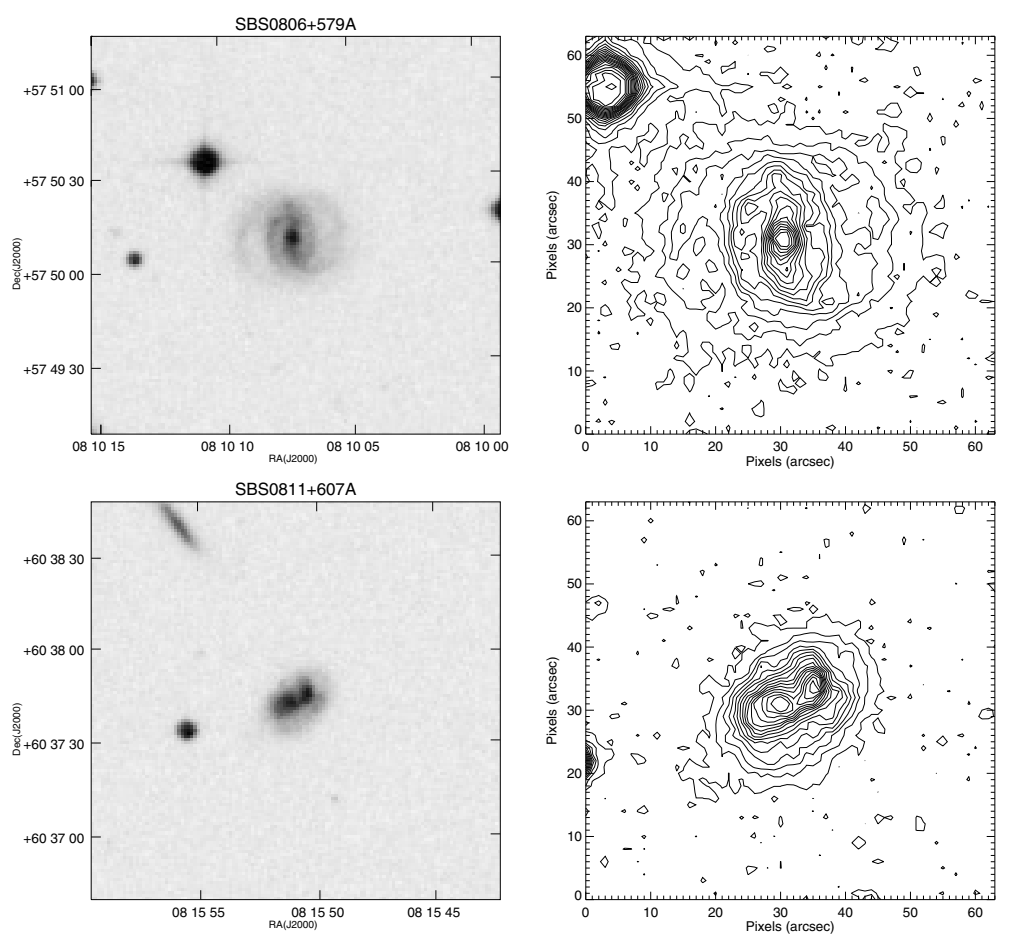

Fig. 1. Grey scale images and isophotal maps of the HI-detected galaxy systems from POSS-II ( $F$-band). The contour levels are in arbitrary units. The lowest contour corresponds to the $3 \sigma$ level of the local background. The scale interval was chosen in order to best illustrate both the inner and outer structure of the galaxies.
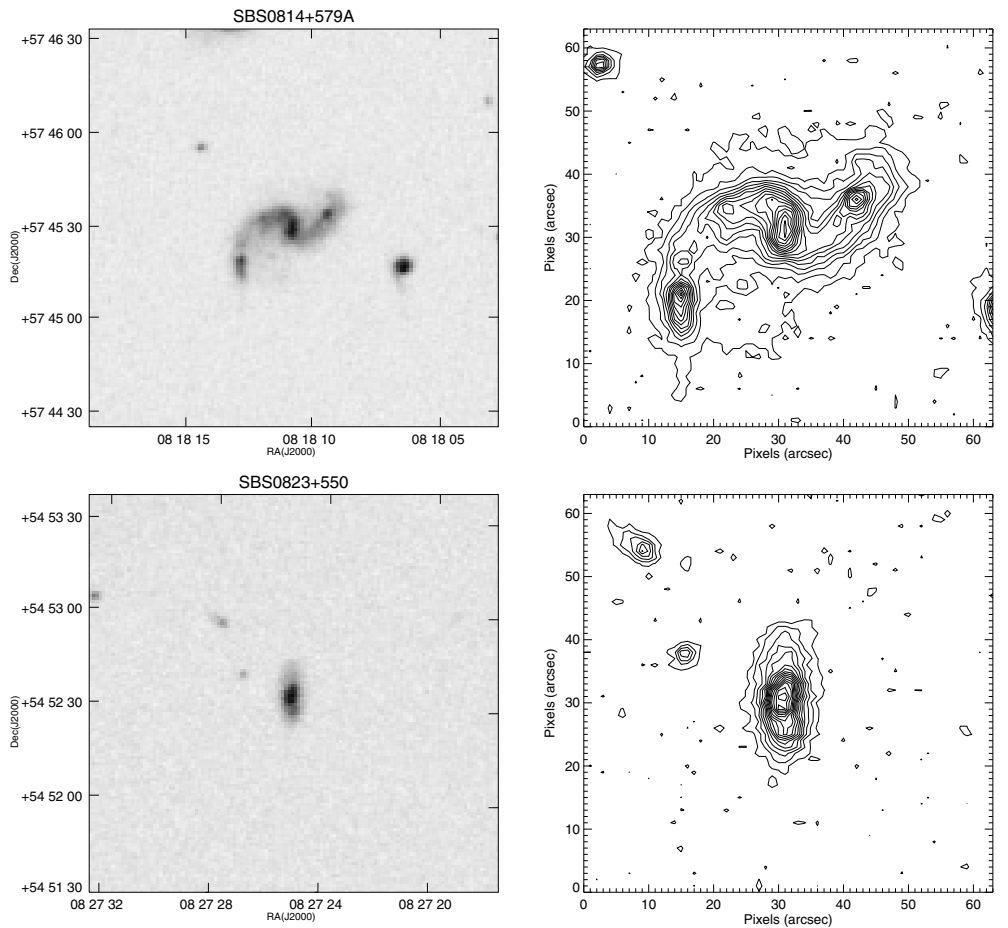

Fig. 1. continued. 
W. K. Huchtmeier et al.: Interacting and merging galaxies. I. (RN), Online Material p 2
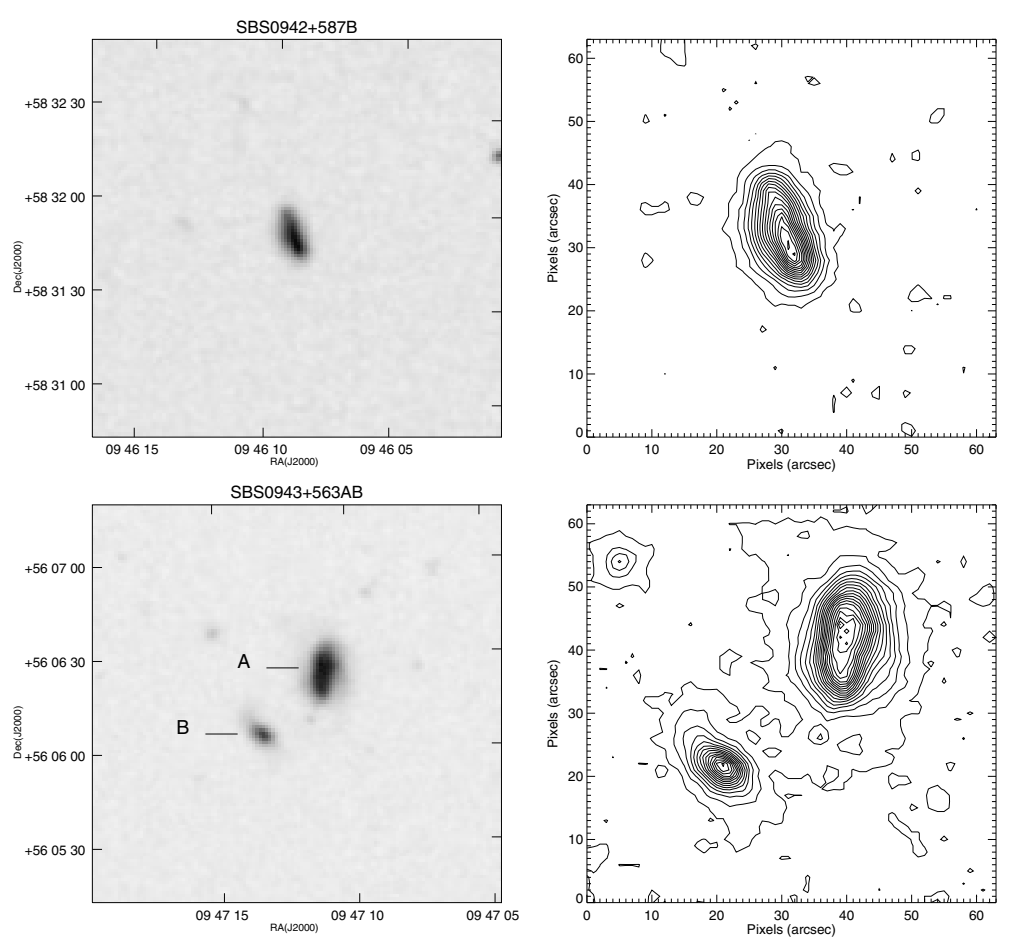

Fig. 1. continued.
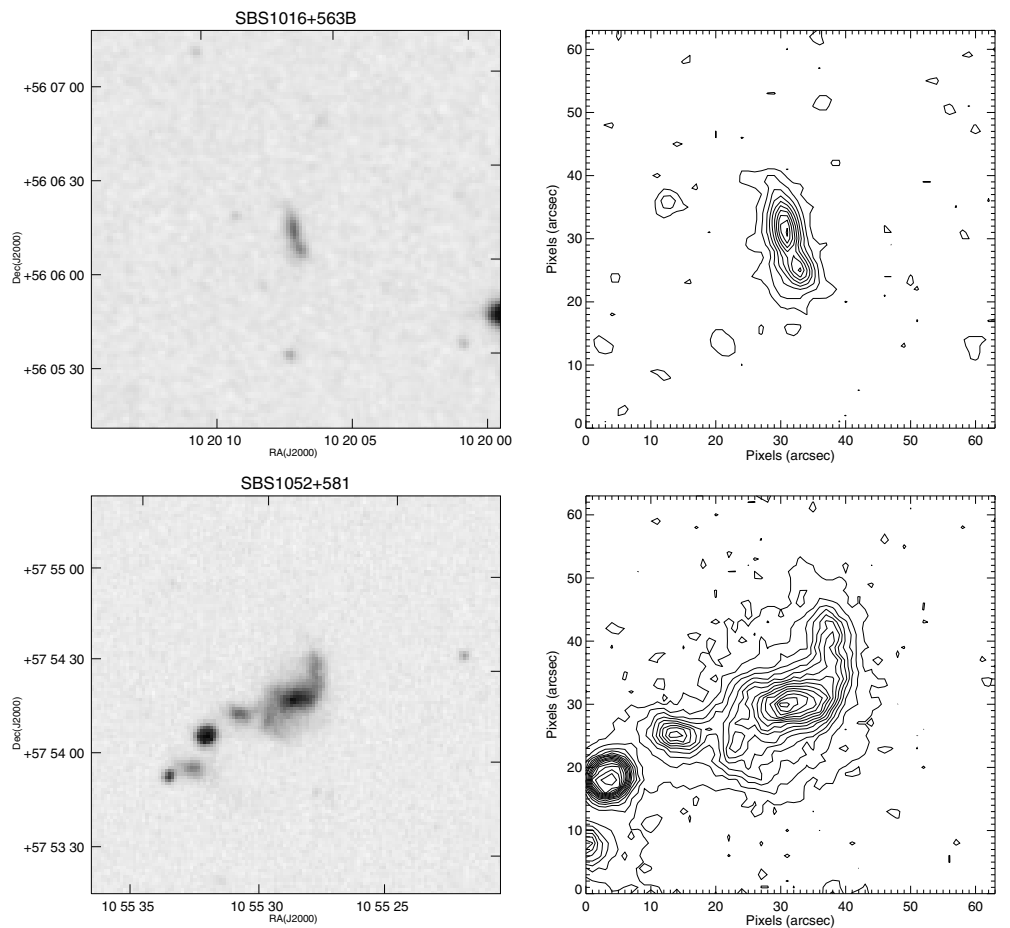

Fig. 1. continued. 
W. K. Huchtmeier et al.: Interacting and merging galaxies. I. (RN), Online Material p 3
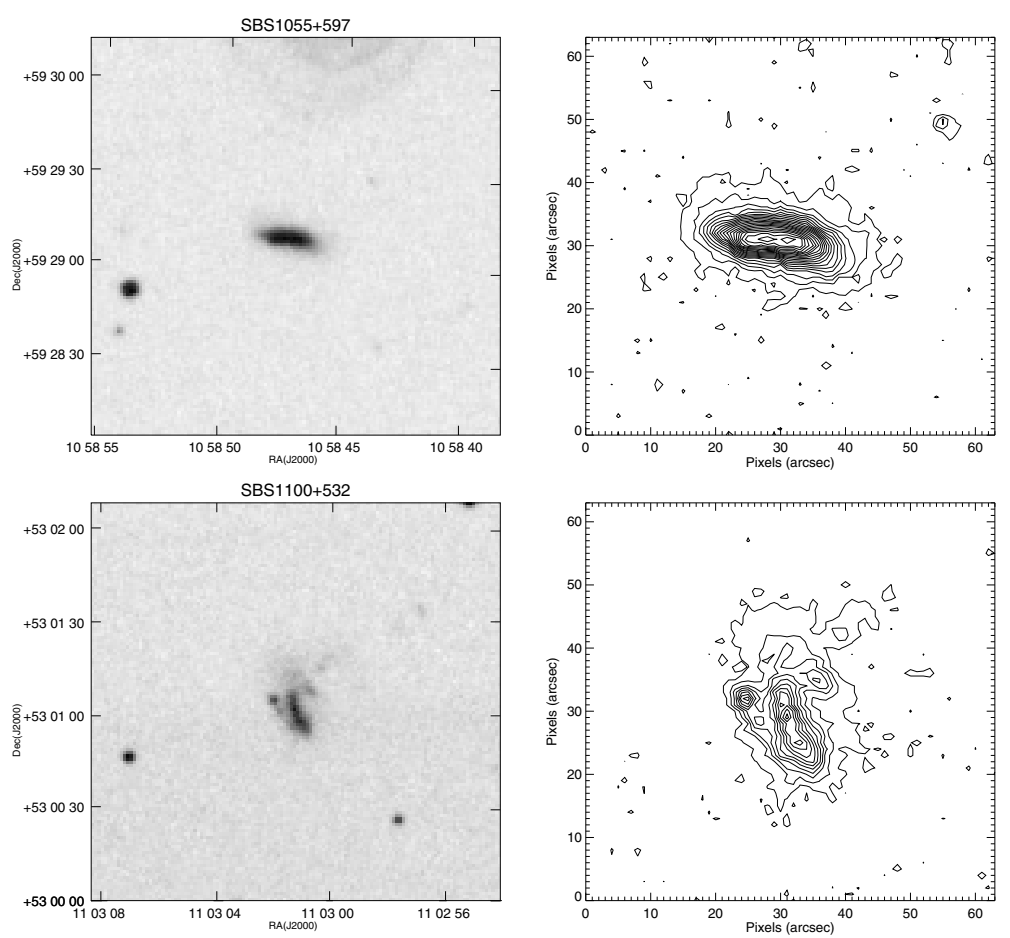

Fig. 1. continued.
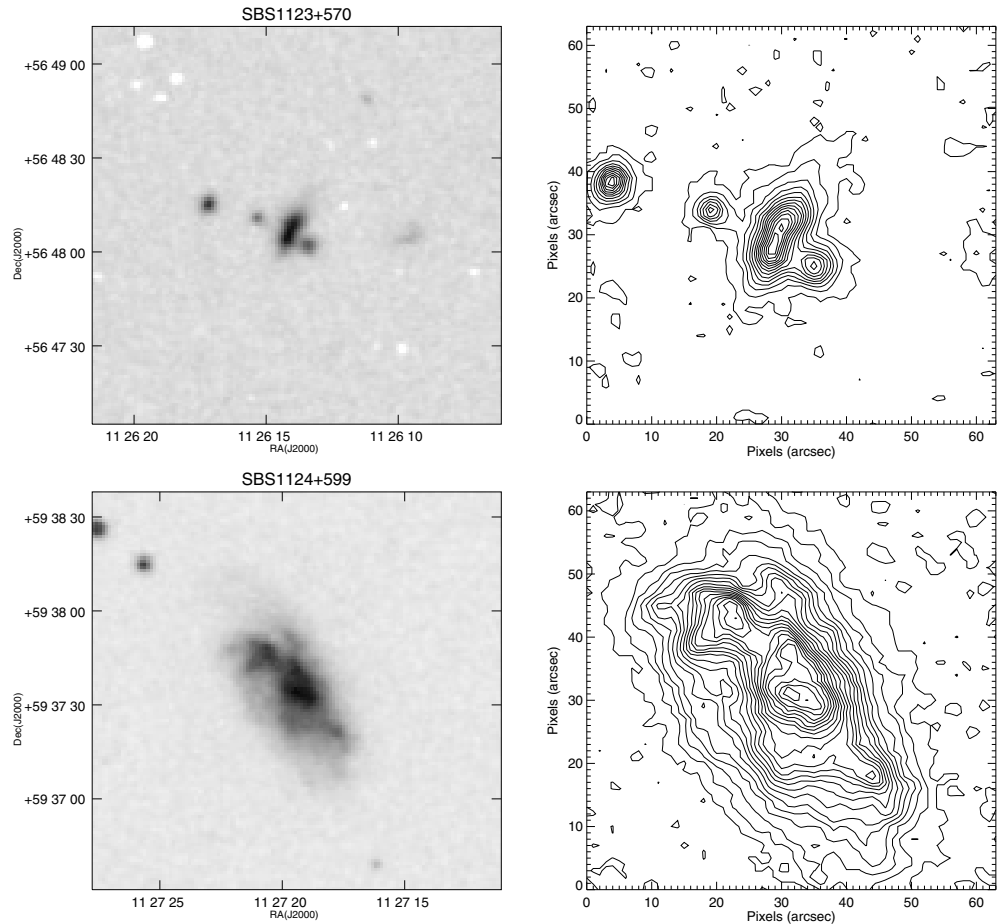

Fig. 1. continued. 
W. K. Huchtmeier et al.: Interacting and merging galaxies. I. (RN), Online Material p 4
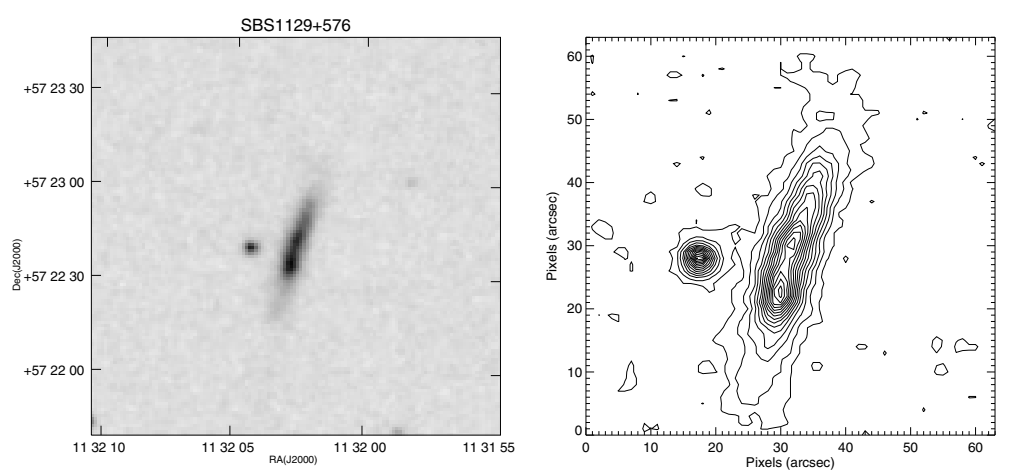

SBS1144+579
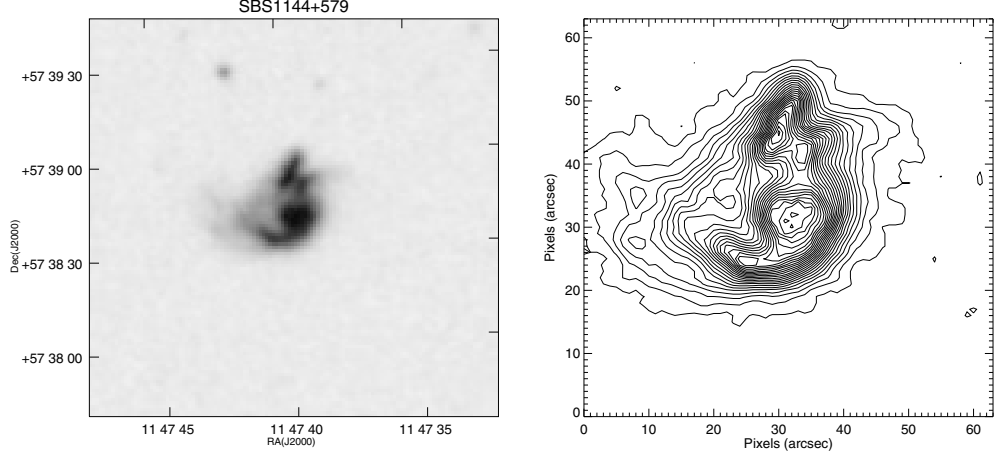

Fig. 1. continued.
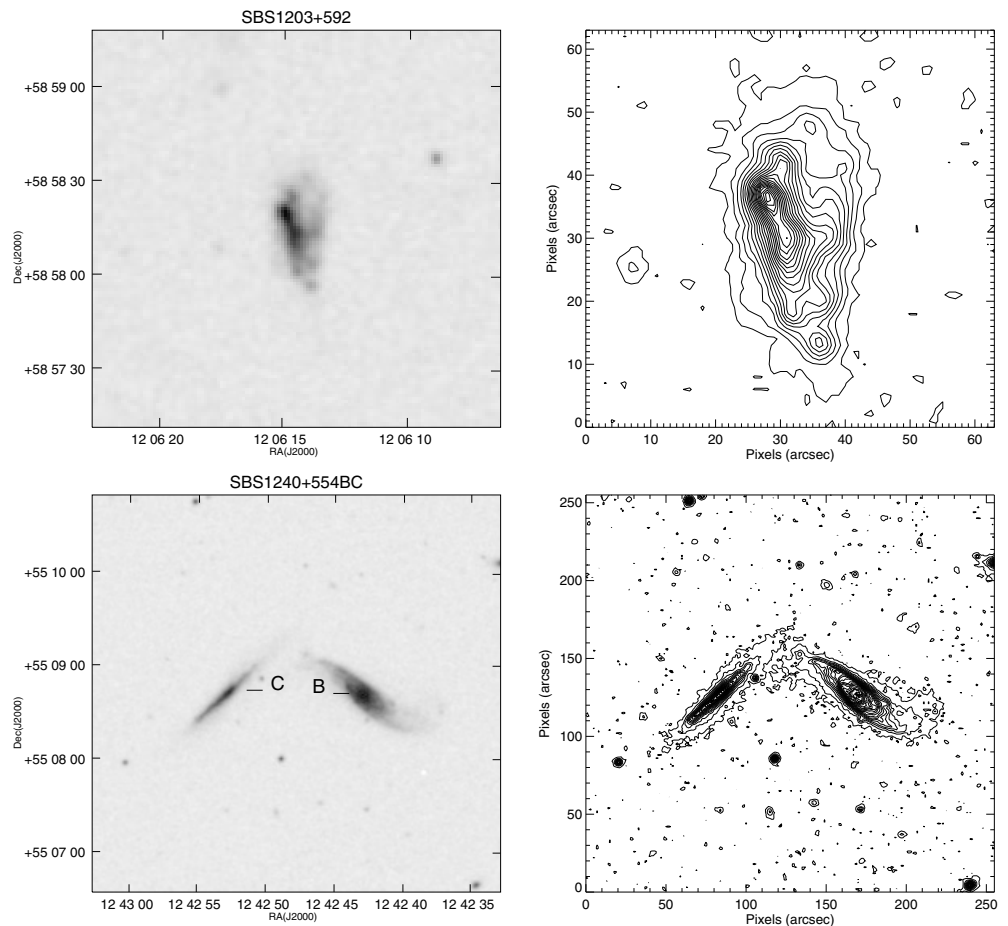

Fig. 1. continued. 
W. K. Huchtmeier et al.: Interacting and merging galaxies. I. (RN), Online Material p 5
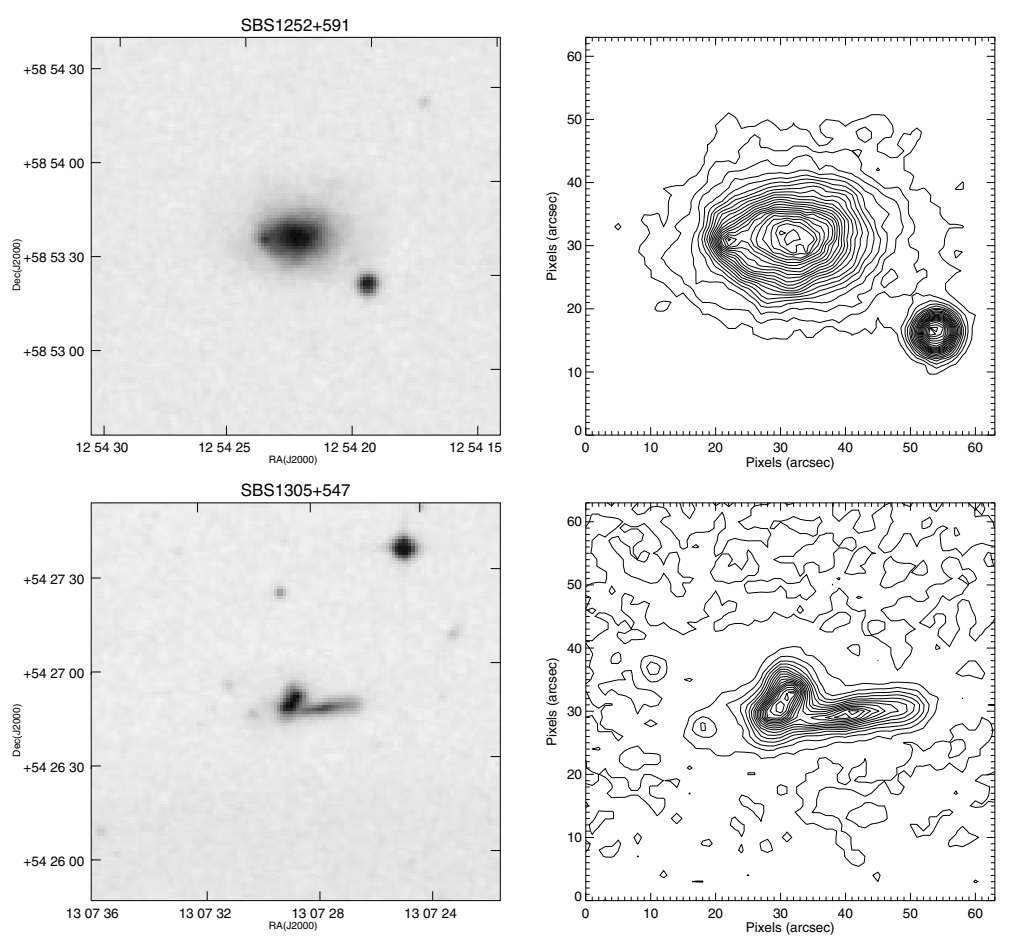

Fig. 1. continued.
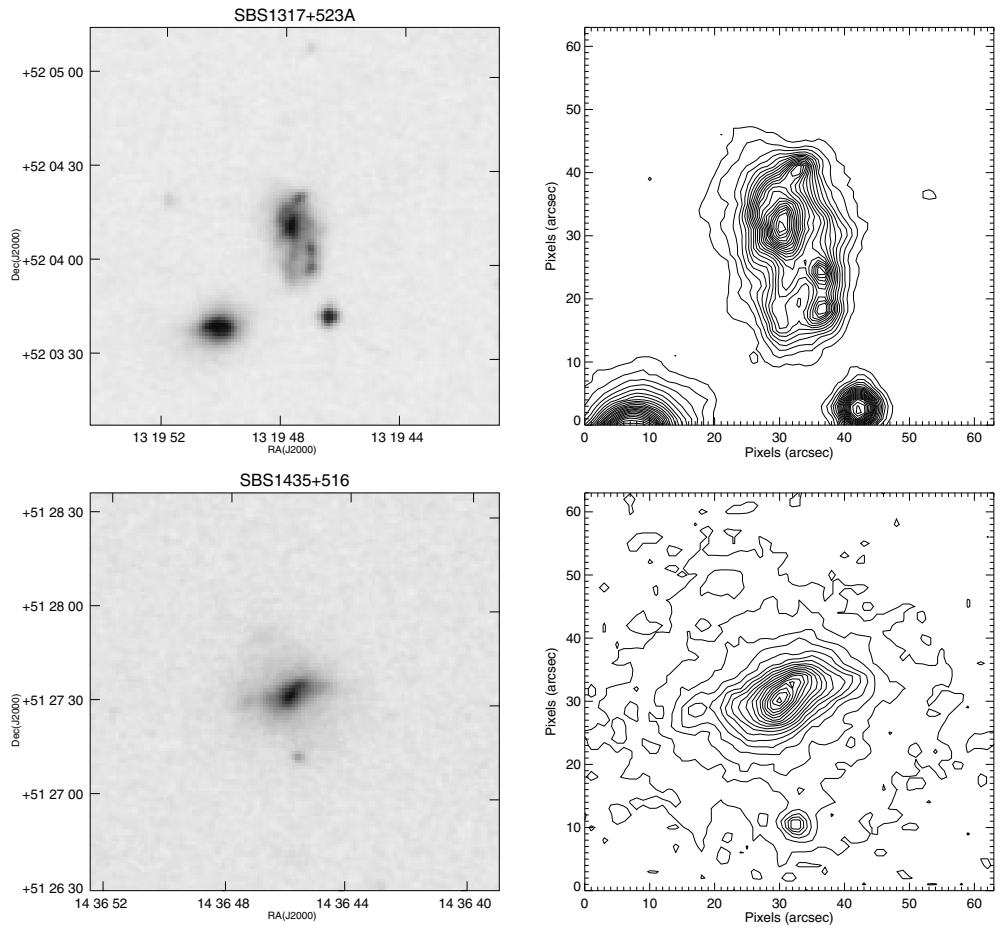

Fig. 1. continued. 
W. K. Huchtmeier et al.: Interacting and merging galaxies. I. (RN), Online Material p 6
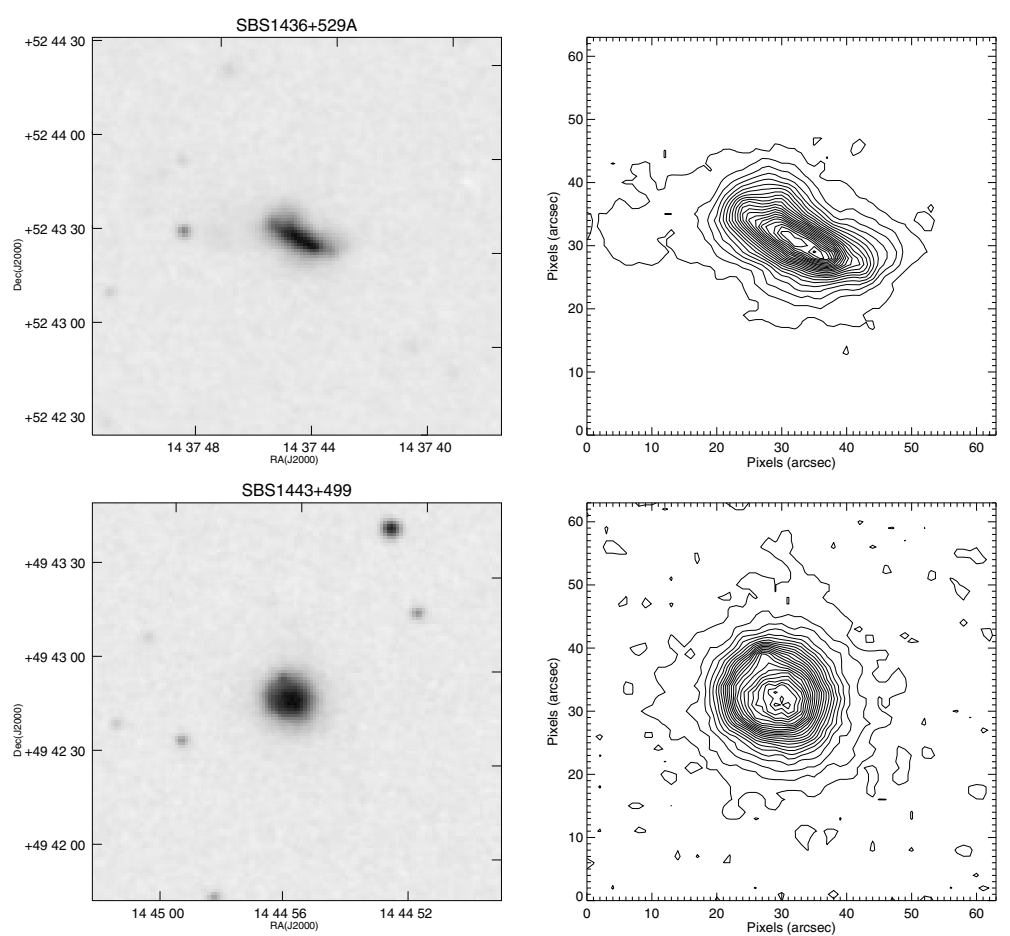

Fig. 1. continued.
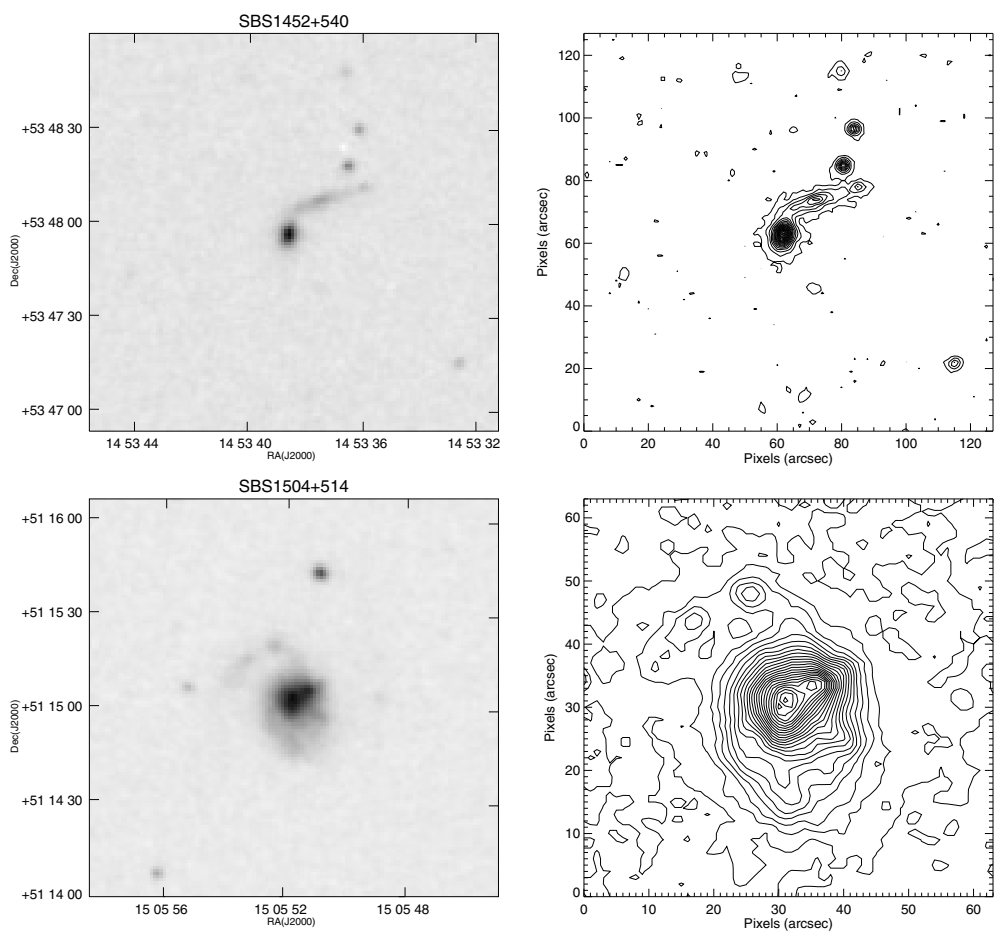

Fig. 1. continued. 
W. K. Huchtmeier et al.: Interacting and merging galaxies. I. (RN), Online Material $p 7$
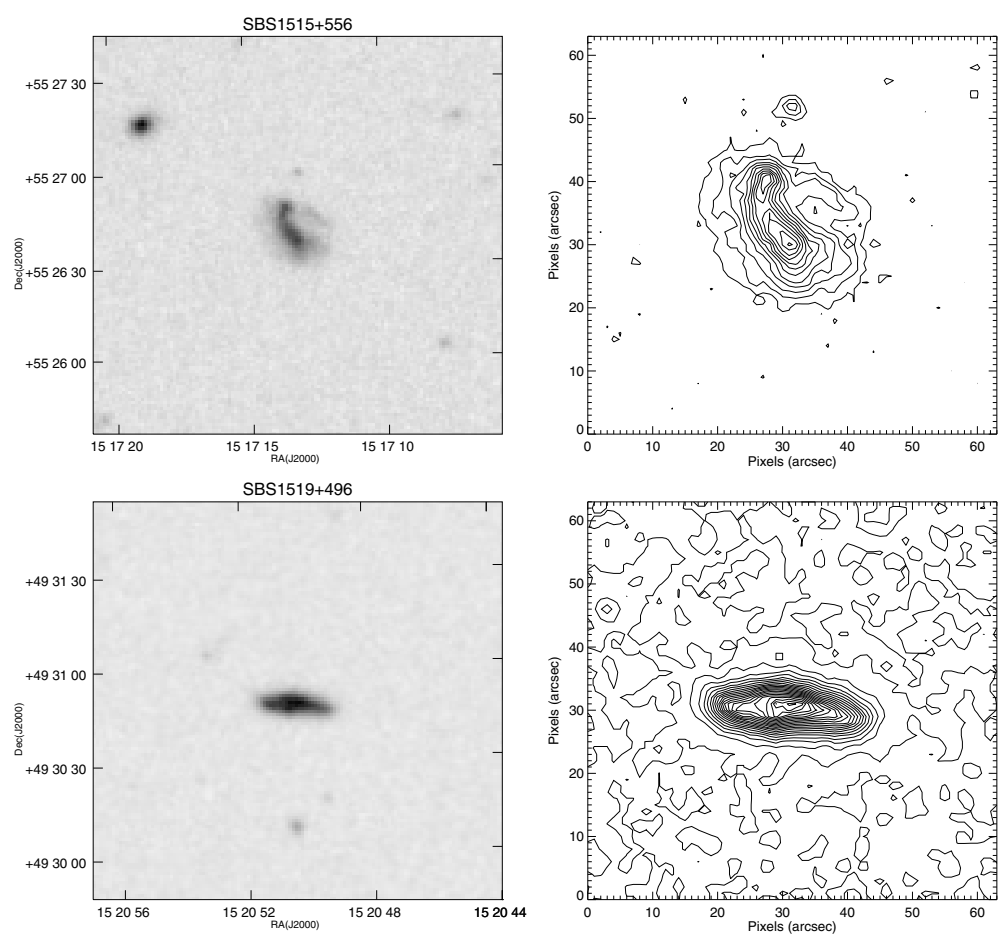

Fig. 1. continued.
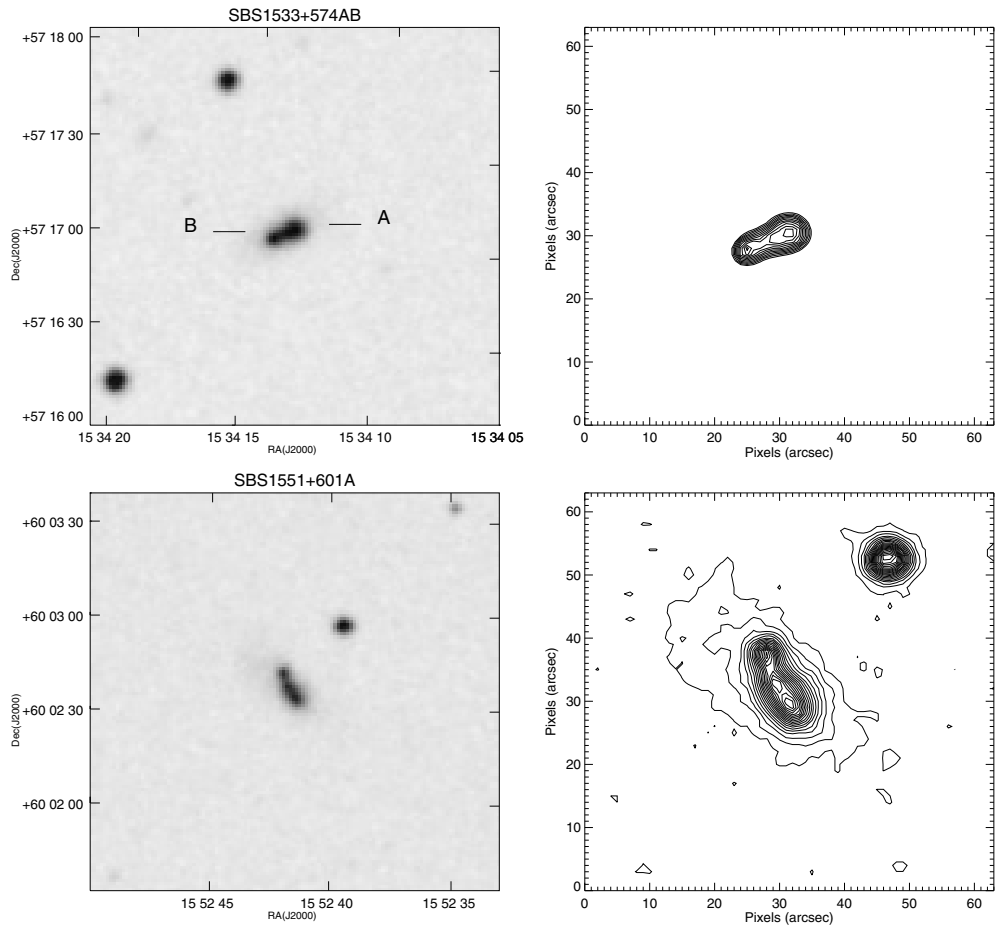

Fig. 1. continued. 
W. K. Huchtmeier et al.: Interacting and merging galaxies. I. (RN), Online Material $p 8$
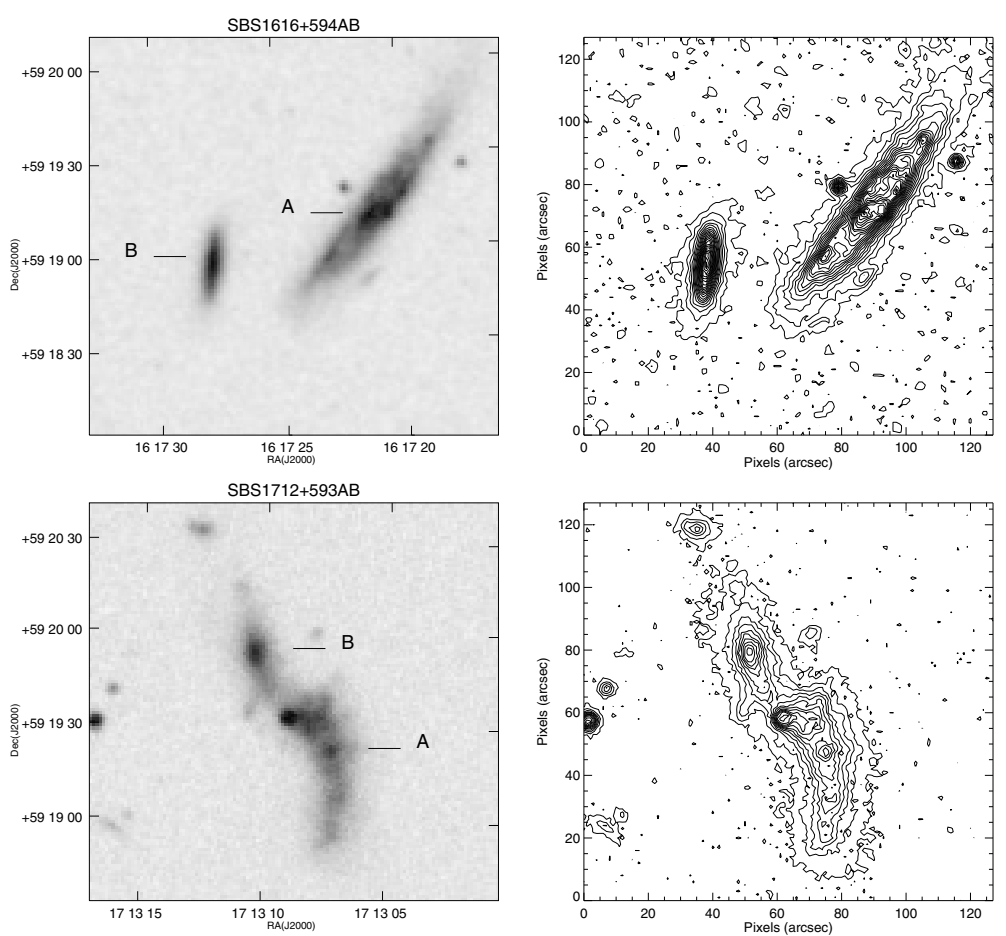

Fig. 1. continued. 
W. K. Huchtmeier et al.: Interacting and merging galaxies. I. (RN), Online Material $p 9$
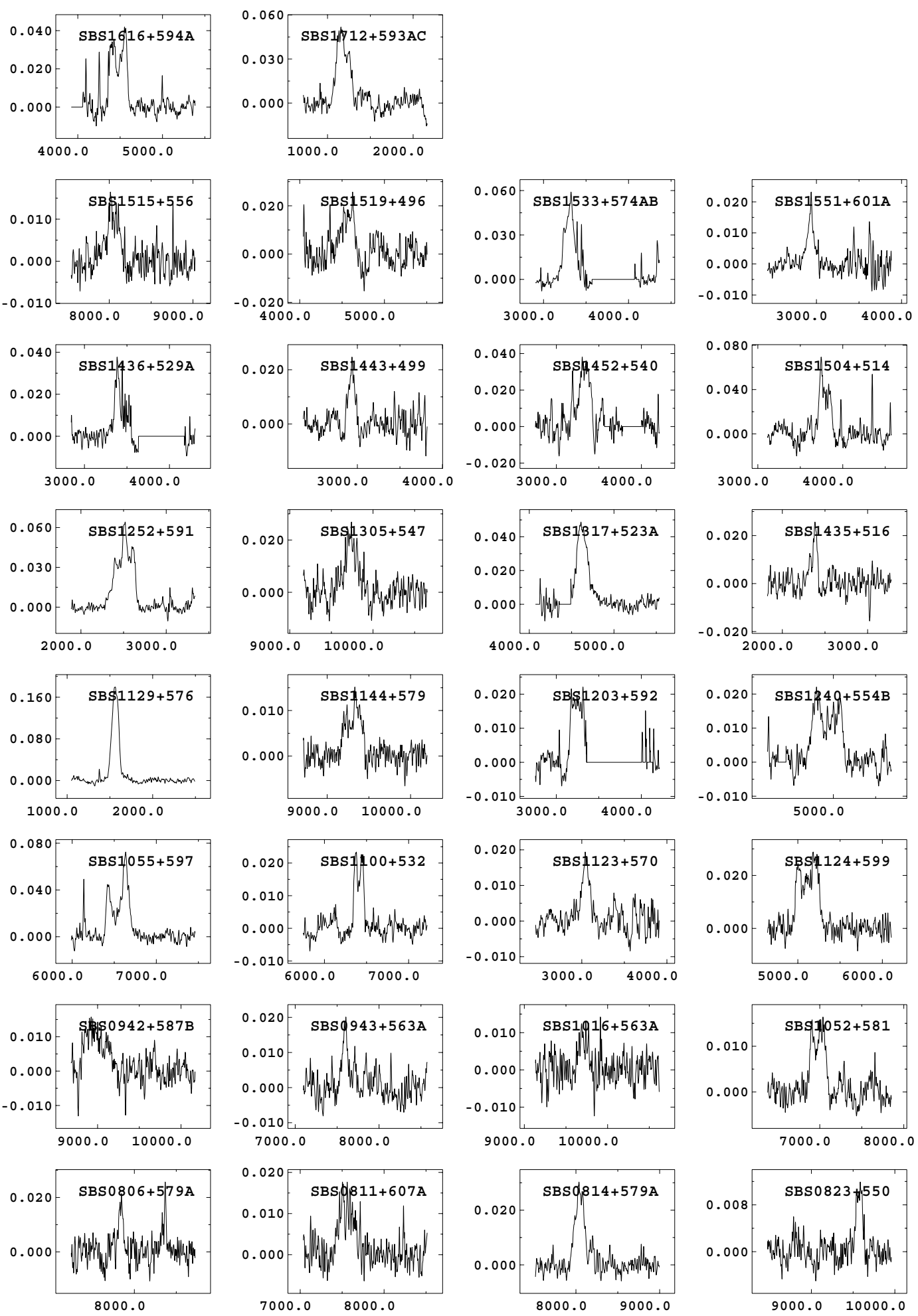

Fig. 2. HI profiles of the 30 SBS galaxies detected in the present Effelsberg observations, with a pencil beam of half-power width of 9.3 arcmin at $21-\mathrm{cm}$. The HI profiles are arranged in an increasing order of right ascension, starting at the bottom left corner (see Table 1). The axes show flux density in Jansky (Jy) and the heliocentric radial velocity in $\mathrm{km} \mathrm{s}^{-1}$. 
W. K. Huchtmeier et al.: Interacting and merging galaxies. I. (RN), Online Material $p 10$

Table 1. Observational data.

\begin{tabular}{|c|c|c|c|c|c|c|c|c|c|}
\hline $\begin{array}{l}\text { Galaxy } \\
\text { name }\end{array}$ & $\mathrm{hms} \circ, "$, & $\begin{array}{l}\text { optical } \\
\text { size } \\
\text { [arcsec] }\end{array}$ & $m_{B}$ & $\begin{array}{r}\text { Opt. } \\
\text { vel. } \\
{\left[\mathrm{km} \mathrm{s}^{-1}\right]}\end{array}$ & $\begin{array}{c}\text { HI-flux } \\
{[\mathrm{Jy}] .} \\
{\left[\mathrm{km} \mathrm{s}^{-1}\right]}\end{array}$ & $\begin{array}{r}\text { HI-Peak } \\
\text { flux } \\
{[\mathrm{mJy}]}\end{array}$ & $\begin{array}{r}\mathrm{HI} \\
\text { velocity } \\
{\left[\mathrm{km} \mathrm{s}^{-1}\right]}\end{array}$ & $\begin{array}{l}\text { HI } \\
\text { linewidth } \\
{\left[\mathrm{km} \mathrm{s}^{-1}\right]}\end{array}$ & $\begin{array}{r}\text { Radio } \\
\text { flux } \\
\text { [mJy] }\end{array}$ \\
\hline SBS 0745+590 & $074916.8+585511$ & 21.4 & 17.5 & 8220 & & \pm 2 & & & $\sim 10$ \\
\hline SBS $0745+587$ & $074936.7+580334$ & 28.6 & 17.0 & 6390 & & \pm 3 & & & \\
\hline SBS 0806+579A & $081007.0+575012$ & 44.9 & 15.1 & 7800 & 1.0 & $21 \pm 3$ & $7841 \pm 6$ & 57 & \\
\hline SBS 0811+607A & $081550.8+603745$ & 33.7 & 15.5 & 7590 & 2.5 & $18 \pm 2$ & $7531 \pm 8$ & 100 & 7.5 \\
\hline SBS 0814+579A & $081810.5+574531$ & 52.0 & 15.3 & 8070 & 3.5 & $30 \pm 2$ & $8040 \pm 8$ & 118 & 5.2 \\
\hline SBS $0823+550$ & $082725.0+555236$ & 24.5 & 16.0 & 9030 & 1.0 & $11 \pm 2$ & $9573 \pm 9$ & 81 & \\
\hline SBS $0830+563$ & $083427.2+560857$ & 28.6 & 17.0 & 7800 & & \pm 3 & & & \\
\hline SBS $0926+558$ & $092956.4+553916$ & 29.6 & 16.0 & 7500 & & \pm 4 & & & \\
\hline SBS $0942+587 B$ & $094608.9+583152$ & 26.5 & 16.5 & 9240 & 3.4 & $17 \pm 3$ & $8951 \pm 6$ & 113 & \\
\hline SBS $0943+563 \mathrm{~A}$ & $094710.7+560627$ & 27.5 & 15.5 & 7650 & 1.4 & $20 \pm 3$ & $7596 \pm 9$ & 59 & $\sim 1.5$ \\
\hline SBS 1000+561 & $100415.9+555052$ & 24.5 & 15.7 & 7650 & & \pm 3 & & & \\
\hline SBS 1016+563A & $102006.6+560614$ & 22.4 & 16.5 & 9690 & 1.2 & $13 \pm 4$ & $9710 \pm 9$ & 90 & \\
\hline SBS 1028+566 & $103207.4+562141$ & 28.6 & 16.5 & 7410 & & \pm 2 & & & \\
\hline SBS $1052+581$ & $105528.6+575422$ & 37.7 & 15.7 & 6930 & 2.5 & $16 \pm 2$ & $6979 \pm 9$ & 197 & $\sim 2.3$ \\
\hline SBS 1055+597 & $105846.9+592912$ & 31.6 & 15.6 & 6540 & 10.8 & $75 \pm 3$ & $6544 \pm 4$ & 267 & $\sim 2.0$ \\
\hline SBS $1100+532$ & $110301.2+530106$ & 29.6 & 16.5 & 6300 & 2.4 & $24 \pm 2$ & $6408 \pm 7$ & 126 & \\
\hline SBS $1123+570$ & $112613.8+564811$ & 26.5 & 17.0 & 3000 & 1.8 & $19 \pm 3$ & $3050 \pm 9$ & 106 & \\
\hline SBS $1124+599$ & $112719.3+593736$ & 87.7 & 14.0 & 5190 & 6.3 & $31 \pm 2$ & $5114 \pm 5$ & 288 & 8.3 \\
\hline SBS $1129+576$ & $113202.5+572248$ & 47.9 & 15.0 & 1590 & 17.5 & $176 \pm 4$ & $1563 \pm 2$ & 91 & \\
\hline SBS 1144+597 & $114739.6+573842$ & 57.1 & 13.9 & 9270 & 2.2 & $14 \pm 2$ & $9317 \pm 9$ & 116 & 15.8 \\
\hline SBS 1146+604 & $114850.0+601144$ & 53.0 & 15.0 & 3510 & & \pm 4 & & & 2.5 \\
\hline SBS $1200+589 B$ & $120322.6+584136$ & 21.4 & 18.5 & 9630 & & \pm 5 & & & \\
\hline SBS 1203+592 & $120614.4+585816$ & 46.9 & 17.0 & 3300 & 3.4 & $24 \pm 3$ & $3246 \pm 9$ & 167 & \\
\hline SBS $1204+591 \mathrm{~A}$ & $120702.2+584959$ & 37.7 & 16.5 & 9480 & & \pm 3 & & & 5 \\
\hline SBS 1208+590 & $121125.0+584532$ & 41.8 & 15.4 & 3270 & & \pm 4 & & & \\
\hline SBS $1240+554 B$ & $124242.8+550843$ & 1018 & 14.8 & 4770 & 5.5 & $23 \pm 3$ & $4926 \pm 9$ & 361 & \\
\hline SBS 1249+493 & $125152.5+490327$ & 24.5 & 18.0 & 7320 & & \pm 4 & & & \\
\hline SBS 1252+591 & $125422.5+585341$ & 58.1 & 15.0 & 2430 & 11.7 & $63 \pm 3$ & $2554 \pm 6$ & 166 & \\
\hline SBS 1301+539 & $130340.7+534324$ & 41.8 & 17.0 & 8400 & & \pm 3 & & & \\
\hline SBS $1305+547$ & $130728.6+542651$ & 44.9 & 16.0 & 9720 & 3.9 & $29 \pm 4$ & $9731 \pm 6$ & 120 & 3.0 \\
\hline SBS $1305+541 \mathrm{~A}$ & $130739.5+535025$ & 34.7 & 16.0 & 9000 & & \pm 4 & & & \\
\hline SBS $1317+523 \mathrm{~A}$ & $131947.5+520420$ & 38.8 & 15.4 & 4620 & 17.9 & $47 \pm 3$ & $4631 \pm 8$ & 327 & 5.5 \\
\hline SBS 1354+580 & $135624.5+574547$ & 23.5 & 17.5 & 8100 & & \pm 3 & & & \\
\hline SBS 1435+516 & $143645.7+512736$ & 49.0 & 15.5 & 2370 & 1.7 & $24 \pm 3$ & $2386 \pm 9$ & 53 & \\
\hline SBS $1436+529 A$ & $143744.8+524334$ & 40.0 & 15.6 & 3390 & 3.0 & $37 \pm 3$ & $3403 \pm 3$ & 95 & \\
\hline SBS $1443+499$ & $144455.9+494250$ & 35.7 & 15.4 & 2910 & 1.5 & $26 \pm 4$ & $2932 \pm 9$ & 72 & \\
\hline SBS $1444+517$ & $144545.1+513451$ & 63.2 & 14.7 & 9270 & & \pm 3 & & & 12.6 \\
\hline SBS $1452+540$ & $145338.4+534759$ & 39.8 & 17.0 & 3300 & 4.9 & $33 \pm 4$ & $3340 \pm 6$ & 142 & \\
\hline SBS $1457+540$ & $145841.3+535129$ & 34.7 & 16.5 & 8100 & & \pm 4 & & & \\
\hline SBS 1504+514 & $150551.6+511506$ & 49.0 & 16.0 & 3660 & 6.7 & $76 \pm 6$ & $3788 \pm 4$ & 137 & $\sim 1.0$ \\
\hline SBS $1509+583 \mathrm{~A}$ & $151016.9+581038$ & 33.7 & 16.5 & 9420 & & $8 \pm 3$ & & & 13 \\
\hline SBS 1510+571 & $151212.6+570008$ & 29.6 & 16.5 & 660 & & \pm 2 & & & \\
\hline SBS $1515+556 \mathrm{~B}$ & $151713.1+552641$ & 29.6 & 16.5 & 8100 & 2.0 & $17 \pm 3$ & $8040 \pm 9$ & 120 & \\
\hline SBS 1519+496 & $152050.2+493050$ & 31.6 & 15.5 & 4590 & 2.5 & $24 \pm 4$ & $4579 \pm 9$ & 130 & \\
\hline SBS 1528+491B & $153015.8+485815$ & 17.3 & 17.0 & 7500 & & \pm 3 & & & \\
\hline SBS $1533+574 \mathrm{AB}$ & $153413.3+571707$ & 33.2 & 14.6 & 3450 & 7.6 & $59 \pm 3$ & $3296 \pm 9$ & 131 & 6.0 \\
\hline SBS $1542+573 B$ & $154348.6+571357$ & 53.0 & 16.0 & 4290 & & \pm 4 & & & \\
\hline SBS $1551+593 B$ & $155211.6+591436$ & 23.5 & 17.0 & 8940 & & \pm 4 & & & \\
\hline SBS 1551+601A & $155241.3+600238$ & 36.7 & 18.5 & 2970 & 1.6 & $23 \pm 3$ & $2931 \pm 9$ & 48 & \\
\hline SBS 1616+594A & $161721.0+591912$ & 109.1 & 14.8 & 4470 & 6.6 & $40 \pm 4$ & $4479 \pm 5$ & 211 & $\sim 7$ \\
\hline SBS 1646+551 & $164725.7+550403$ & 17.3 & 16.5 & 5280 & & \pm 3 & & & \\
\hline SBS 1657+590A & $165831.7+585613$ & 78.5 & 14.6 & 5490 & & \pm 4 & & & 149 \\
\hline SBS $1712+593 \mathrm{AB}$ & $171308.6+591940$ & 68.4 & 16.5 & 1260 & 7.8 & $52 \pm 5$ & $1182 \pm 5$ & 171 & \\
\hline
\end{tabular}


W. K. Huchtmeier et al.: Interacting and merging galaxies. I. (RN), Online Material $p 11$

Table 2. Derived parameters for the detected galaxies.

\begin{tabular}{|c|c|c|c|c|c|c|c|c|c|c|}
\hline $\begin{array}{l}\text { Galaxy } \\
\text { name } \\
\text { (SBS) }\end{array}$ & $\begin{array}{r}V_{3 \mathrm{Kbgd}} \\
{\left[\mathrm{km} \mathrm{s}^{-1}\right]}\end{array}$ & $\begin{array}{r}\text { Dist. } \\
D \\
\mathrm{Mpc}\end{array}$ & $\begin{array}{r}\text { Diam. } \\
A_{0, i} \\
\mathrm{kpc}\end{array}$ & $\begin{array}{c}\text { Abs. } \\
\text { mag. } \\
M_{B}\end{array}$ & $\begin{array}{r}\mathrm{HI} \\
\text { mass } \\
{\left[10^{9} M_{\odot}\right]}\end{array}$ & $\begin{array}{r}\text { Total } \\
\text { mass } \\
{\left[10^{10} M_{\odot}\right]}\end{array}$ & $\begin{array}{r}M_{\mathrm{HI}} / L_{B} \\
{\left[M_{\odot} / L_{\odot}\right]}\end{array}$ & $\begin{array}{r}M_{\mathrm{T}} / L_{B} \\
{\left[M_{\odot} / L_{\odot}\right]}\end{array}$ & $\begin{array}{c}M_{\mathrm{HI}} / M_{\mathrm{T}} \\
{\left[M_{\odot} / L_{\odot}\right]}\end{array}$ & Comments \\
\hline $0806+579 A$ & 7894 & 108 & 23.9 & -20.28 & 2.8 & & & & & \\
\hline $0811+607 \mathrm{~A}$ & 7659 & 105 & 17.2 & -20.06 & 6.5 & 3.4 & 0.4 & 2.0 & 0.19 & \\
\hline 0814+579A & 8152 & 112 & 26.1 & -20.21 & 10.3 & & & & & \\
\hline $0823+550$ & 9362 & 128 & 13.8 & -19.75 & 3.9 & & & & & \\
\hline 0942+587B & 9374 & 128 & 14.9 & -19.25 & 13.2 & & & & & \\
\hline $0943+563 B$ & 7732 & 106 & 8.1 & -19.69 & 5.3 & & & & & \\
\hline $1016+563 A$ & 9853 & 137 & 13.0 & -19.35 & 5.3 & 1.4 & 0.62 & 1.7 & 0.37 & \\
\hline $1052+581$ & 7912 & 97 & 15.8 & -19.62 & 5.6 & & & & & \\
\hline $1055+597$ & 7082 & 97 & 13.1 & -19.87 & 23.9 & & & & & \\
\hline $1100+532$ & 6602 & 90 & 11.8 & -18.49 & 4.6 & & & & & \\
\hline $1123+570$ & 3220 & 44 & 5.7 & -16.31 & 0.8 & & & & & \\
\hline $1124+599$ & 5259 & 72 & 27.8 & -20.89 & 7.7 & & & & & \\
\hline $1129+576$ & 1726 & 24 & 4.3 & -16.98 & 2.3 & 0.4 & 2.38 & 4.3 & 0.56 & \\
\hline $1144+579$ & 9379 & 128 & 33.5 & -21.83 & 8.6 & & & & & \\
\hline $1203+592$ & 3402 & 47 & 9.4 & -16.76 & 1.7 & & & & & \\
\hline $1240+554 \mathrm{~B}$ & 5094 & 70 & 28.8 & -20.44 & 6.3 & & & & & \\
\hline $1252+591$ & 2583 & 35 & 9.6 & -17.99 & 3.4 & & & & & \\
\hline $1305+547$ & 9864 & 135 & 24.6 & -19.95 & 16.8 & 4.4 & 1.13 & 3.0 & 0.38 & \\
\hline $1317+523 \mathrm{~A}$ & 4775 & 65 & 11.5 & -19.12 & 18.0 & & & & & \\
\hline $1435+516$ & 2490 & 34 & 8.0 & -17.22 & 0.5 & 0.8 & 0.39 & 6.5 & 0.06 & \\
\hline $1436+529 \mathrm{~A}$ & 3487 & 48 & 8.6 & -17.92 & 1.6 & 1.4 & 0.70 & 6.2 & 0.11 & \\
\hline 1443+499 & 3053 & 42 & 7.3 & -17.85 & 0.6 & & & & & \\
\hline $1452+540$ & 3438 & 47 & 7.3 & -16.79 & 2.6 & & & & & \\
\hline $1504+514$ & 3881 & 53 & 12.2 & -17.84 & 4.5 & & & & & \\
\hline $1515+556$ & 8123 & 111 & 15.2 & -18.92 & 5.8 & 5.0 & 1.0 & 8.6 & 0.12 & \\
\hline $1519+496$ & 4620 & 63 & 8.4 & -18.80 & 2.4 & 1.9 & 0.46 & 3.8 & 0.12 & \\
\hline $1533+574 \mathrm{~A}$ & 3393 & 46 & 4.9 & -18.91 & 3.9 & & & & & \\
\hline $1551+601 \mathrm{~A}$ & 2955 & 40 & 6.4 & -14.60 & 0.6 & & & & & \\
\hline $1616+594 \mathrm{~A}$ & 4477 & 61 & 26.3 & -19.89 & 5.8 & 12.9 & 0.42 & 9.4 & 0.05 & \\
\hline $1712+593 \mathrm{AB}$ & 1131 & 16 & 4.6 & -14.88 & 0.4 & & & & & \\
\hline
\end{tabular}




\section{Appendix A: Comments on individual galaxies}

Since, for each galaxy, the $21 \mathrm{~cm}$ receiver was tuned to the frequency corresponding to its optical radial velocity, its HI profile should appear near the center of its panel in Fig. 1. A substantially off-centred profile is likely to indicate confusion or blending with $\mathrm{HI}$ emission from other galaxies within the radio telescope beam.

Around the position of each galaxy in our sample, we scrutinized a region of 9.3 arcmin radius (i.e., a region twice the half-power beamwidth of the Effelsberg telescope) on the Digital Sky Survey (DSS), also paying attention to the velocity and other data provided in the NED (NASA/IPAC Extragalactic Database). Based on this, if we failed to identify one or more likely sources of confusion for the observed HI profile, as described below, we accepted the HI profile as being wholly associated with the target galaxy.

SBS 0745+590 - The radio counterpart consists of an unresolved component at $074918.7+585519$ (J2000), with a peak flux of $4.0 \pm 0.4 \mathrm{mJy}$ at $1.4 \mathrm{GHz}$ and $\sim 1^{\prime}$ long extension at PA $60 \mathrm{deg}$ (total flux $\sim 10 \mathrm{mJy}$ ).

SBS 0806+579A - This target galaxy of type Sc has confusion candidates at similar velocities. Also, it forms a physical pair with a UV-excess object, the Sb type galaxy SBS 0806+579B separated by $1.55 \mathrm{arcmin}$. The third candidate for confusion, VII Zw215 $\left(8284 \mathrm{~km} \mathrm{~s}^{-1}\right)$, is offset in radial velocity by $\sim 500 \mathrm{~km} \mathrm{~s}^{-1}$ and a corresponding very narrow HI profile is hinted at in Fig. 1.

SBS 0811+607A - A merging system with double nuclei separated by 5 arcsec. This galaxy forms a physical pair with SBS 0811+607B $\left(V_{\text {hel }}=7315 \mathrm{~km} \mathrm{~s}^{-1}\right)$ lying 1.14 arcmin (35 kpc) away. Component B appears edge-on. A broad HI-profile centered at $7531 \mathrm{~km} \mathrm{~s}^{-1}$ corresponds to A. No HI is detected at the optical velocity of component $\mathrm{B}\left(7315 \mathrm{~km} \mathrm{~s}^{-1}\right)$ which, however, has a large error. No known confusing object exist.

The radio counterpart consists of an unresolved component at $081550.9+603743(\mathrm{~J} 2000)$, with a flux of $7.5 \pm 0.5 \mathrm{mJy}$ and $\mathrm{a} \sim 1.5^{\prime}$ long faint extension at PA $\sim 30 \mathrm{deg}$.

SBS 0814+579A - SBc type spiral galaxy with a Sy3 nucleus, in possible interaction with a dwarf companion projected on its south-eastern spiral arm. The two objects are separated by $20.5 \operatorname{arcsec}(11 \mathrm{kpc})$. Another SBS object SBS 0814+579C $\left(V_{\text {hel }}=8094 \mathrm{~km} \mathrm{~s}^{-1}\right)$, an SBb type spiral galaxy with a Sy3 nucleus, is separated by $1.2 \operatorname{arcsec}(0.6 \mathrm{kpc})$ from component A. This pair of galaxies has been reported by Thuan et al. (1999) to be confused.

The radio counterpart is marginally resolved (size $\sim 45$ arcsec), with a centroid at $081811.1+574524$ (J2000), a peak flux of $2.9 \pm 0.4 \mathrm{mJy}$ and integrated flux of $5.2 \pm 0.9 \mathrm{mJy}$.

SBS 0823+550 - Double nuclei undergoing a merger; its outer structure resembles a spiral galaxy. The two nuclei are separated by $1.5 \operatorname{arcsec}(0.9 \mathrm{kpc})$. This object forms a triplet, with the S0 type galaxy PGC023712 $\left(V_{\mathrm{hel}}=9163 \mathrm{~km} \mathrm{~s}^{-1}\right.$, separated by 1.89 arcmin $(70 \mathrm{kpc})$ to the north) and with the spiral galaxy SDSS J082723.89+545045,9 $\left(V_{\mathrm{hel}}=9608 \mathrm{~km} \mathrm{~s}^{-1}\right.$; separated by 1.85 arcmin $(69 \mathrm{kpc})$ to the south).

The HI peak $\left(9578 \mathrm{~km} \mathrm{~s}^{-1}\right)$ is probably associated with SDSS J082723.89+545045 (9608 $\left.\mathrm{km} \mathrm{s}^{-1}\right)$.

SBS 0942+587 B - A merger having double nuclei 3.2 arcsec $(2 \mathrm{kpc})$ apart. This system forms a physical pair with the Sc type galaxy SBS $0942+587$ A separated by 2.12 arcmin $(79 \mathrm{kpc})$ to the southwest.

The broad HI line $\left(V_{\text {hel }}=8927 \mathrm{~km} \mathrm{~s}^{-1}\right)$ corresponds to the optical velocities of one of the companions of SBS $0942+587 \mathrm{~B}$ $\left(8975 \mathrm{~km} \mathrm{~s}^{-1}\right)$ and the galaxy SBS 0942+587 A $\left(9054 \mathrm{~km} \mathrm{~s}^{-1}\right)$ located at $09 \mathrm{~h} 45 \mathrm{~m} 54.7 \mathrm{~s}+58^{\circ} 30^{\prime} 56^{\prime \prime}$ (J2000).

SBS 0943+563 A=Mrk123 - This SB0/a type galaxy forms a close physical pair with the blue compact galaxy (BCG) SBS 0943+563B $\left(V_{\text {hel }}=7578 \mathrm{~km} \mathrm{~s}^{-1}\right)$ located at a angular separation of $27.5 \operatorname{arcsec}(14 \mathrm{kpc})$. The observed HI peak $\left(7596 \mathrm{~km} \mathrm{~s}^{-1}\right)$ corresponds to the optical velocities of components $\mathrm{A}$ and $\mathrm{B}$. No other confusing object is obvious.

A faint radio counterpart $(\sim 1.5 \mathrm{mJy})$ is seen at $094712.4+560608(\mathrm{~J} 2000)$.

SBS 1016+563 A - A merger having two nuclei 6.2 arcsec (4 kpc) apart. Its outer structure resembles a spiral galaxy. The brightest nucleus in the system is an AGN with possible Sy3 characteristics.

The weak HI profile may have some contribution from SBS $1016+563 \mathrm{~B}\left(9717 \mathrm{~km} \mathrm{~s}^{-1}, 6.3^{\prime}(250 \mathrm{kpc}) \mathrm{NE}\right)$ and $2 \mathrm{MASX}$ $\mathrm{J} 10200806+5607557\left(9820 \mathrm{~km} \mathrm{~s}^{-1}\right)$.

SBS 1052+581 - An SBc type spiral galaxy with a starburst nucleus. This galaxy is interacting with a fainter, possibly spiral, galaxy offset towards the east by $18.7 \operatorname{arcsec}(9 \mathrm{kpc})$. Another possibly spiral galaxy with a similar brightness lies to the southeast, separated by 40.6 arcsec ( $19 \mathrm{kpc}$ ) from the SBS galaxy.

The optical image shows strong tidal deformation. There is no obvious confusion in the HI profile which appears to correspond to a disk-like galaxy.

A faint radio counterpart $(\sim 2.3 \pm 0.4 \mathrm{mJy})$ is seen at $105529.1+575430(\mathrm{~J} 2000)$.

SBS 1055+597 - A merger system with two nuclei 6.4 arcsec apart. Its outer structure resembles a spiral galaxy. This galaxy is also known as the high surface brightness object Akn271 and is a member of the isolated galaxy pair KPG259B. It is separated from the other member KPG259A=NGC3470 $\left(V_{\text {hel }}=6548 \mathrm{~km} \mathrm{~s}^{-1}\right)$ by $1.46 \operatorname{arcmin}(41 \mathrm{kpc})$ NW. KPG269A, a Sab galaxy, was detected in HI by Theureau et al. (1998). Van den Bergh et al. (2005) detected the SN2004a in this galaxy.

The asymmetric double-horn HI profile is probably largely due to the confusing spiral galaxy NGC 3470 (see above). Another potential confusing source, albeit to a much less degree, is SDSS J1010575.63+59291 $\left(6711 \mathrm{~km} \mathrm{~s}^{-1}\right)$.

A faint radio counterpart $(\sim 2 \mathrm{mJy})$ is seen at $105846.3+592906(\mathrm{~J} 2000)$.

SBS 1100+532 - Possible merger - could even be an irregular galaxy. In its center, two unequally bright condensations have been detected at a separation of $1.6 \operatorname{arcsec}(0.7 \mathrm{kpc})$. Two other elongated faint compact features are associated with the central region of this sytem.

SBS 1123+570 - A merger system, having two nuclei separated by 3.6 arcsec. The central body of the system is connected to a diffuse object 7.9 arcsec to the west and a diffuse tail to the relatively higher surface brightness object at a distance of $11.3 \operatorname{arcsec}(2.4 \mathrm{kpc})$ to the east.

No confusion candidate is obvious from NED. There is a galaxy cluster in the background. Also, there are three foreground galaxies offset by $3.7^{\prime}\left(1496 \mathrm{~km} \mathrm{~s}^{-1}\right), 6.3^{\prime}\left(2358 \mathrm{~km} \mathrm{~s}^{-1}\right)$ and $7.6^{\prime}\left(2471 \mathrm{~km} \mathrm{~s}^{-1}\right)$.

SBS 1124+599 - A close interacting pair consisting of a latetype spiral galaxy and a disturbed dwarf 13.8 arcsec to the northeast. The system of UGC 06452 was observed previously in the 
$21 \mathrm{~cm}$ line, its HI flux is $6.5 \mathrm{Jy} \mathrm{km} \mathrm{s}^{-1}$ (Richter \& Huchtmeier 1991), in good agreement with the present (considerably more sensitive) observations.

A marginally resolved radio counterpart of $8.3 \pm 0.1 \mathrm{mJy}$ is located at $112719.0+593739$ (J2000).

SBS 1129+576 - A strong single-peaked HI profile. It is probably largely due to SBS $1129+577$, a bright face-on spiral $3.5^{\prime}(24 \mathrm{kpc}) N\left(1566 \mathrm{~km} \mathrm{~s}^{-1}\right)$. Earlier HI observations are reported by Huchtmeier et al. (2005). Ekta et al. (2006) have mapped the system with the GMRT.

SBS 1144+579 - At least two interacting galaxies with strong tidal tails. An Sb spiral galaxy and an elongated (spiral?) galaxy lying to the north and having a double nucleus, form the isolated galaxy pair KPG301 A and B separated by 13.1 arcsec $(8 \mathrm{kpc})$. The two nuclei in KPG301B are separated by 1.6 arcsec $(1 \mathrm{kpc})$. A compact object seen between KPG301A and B is separated from the southern spiral galaxy by $10.7 \operatorname{arcsec}(6.6 \mathrm{kpc})$. The HI profile is of the double-horn type. No other obvious confusion candidates are seen within the telescope beam.

A $15.8 \pm 0.4 \mathrm{mJy}$ unresolved radio counterpart lies at 1147 $39.7+573848$ (J2000).

SBS 1146+604 - A $\sim 2.5 \mathrm{mJy}$ marginally resolved radio counterpart lies at $114849.9+601139$ (J2000).

SBS 1203+592 - A merger of two nuclei separated by 6.9 arcsec. With an absolute magnitude of -16.6 , this galaxy can be classified as a blue compact dwarf. In that case the two nuclei could be giant HII regions. According to Thuan et al. (1999) the $\mathrm{HI}$ emission of this galaxy amounts to $4.05 \pm 0.60 \mathrm{Jy} \mathrm{km} \mathrm{s}^{-1}$, in agreement with the present observation.

The HI profile could have minor contributions arising from two faint nearby galaxies offset by $1.4^{\prime}(19 \mathrm{kpc})\left(3394 \mathrm{~km} \mathrm{~s}^{-1}\right)$, and $4.6^{\prime}(63 \mathrm{kpc})\left(3710 \mathrm{~km}^{-1}\right)$.

SBS 1204+591A - A faint radio counterpart of size $\sim 1.5^{\prime}$ at $\mathrm{PA} \sim 155 \mathrm{deg}$., a peak flux of $1.7 \pm 0.5 \mathrm{mJy} / \mathrm{beam}$ and total flux of $5 \pm 2$ mJy lies at $120703.1+584946($ J2000).

SBS 1240+554 - A Sb-type spiral galaxy with a starburst nucleus forms a pair with this edge-on disk galaxy SBS $1240+554 \mathrm{C}$ having an HII nucleus $\left(V_{\text {hel }}=4800 \mathrm{~km} \mathrm{~s}^{-1}\right)$. The separation between these two galaxies (KPG352A and B) is $1.43 \operatorname{arcmin}(29 \mathrm{kpc})$. Bottinelli et al. (1999) reported an HI flux of $3.9 \pm 1.5 \mathrm{Jy} \mathrm{km} \mathrm{s}^{-1}$, in agreement with the present work.

NGC $4644 \mathrm{~B}$ at $1.4^{\prime}(28 \mathrm{kpc})$ separation $\left(4808 \mathrm{~km} \mathrm{~s}^{-1}\right)$ is an interacting system. The double-peaked HI profile represents both the interacting galaxies. No other obvious confusing source is found within the Effelsberg beam.

SBS1252+591 - Merger of an Sc-type spiral galaxy having an HII nucleus, with a compact blue object located 8.9 arcsec $(1.5 \mathrm{kpc})$ away. A number of galaxies seen within the telescope beam could be contributing to the multi-peaked HI profile. The three brightest galaxies among them are offset from SBS1252+591 by $3.6^{\prime}(36 \mathrm{kpc})\left(2580 \mathrm{~km} \mathrm{~s}^{-1}\right), 7.6^{\prime}(77 \mathrm{kpc})$ $\left(2515 \mathrm{~km} \mathrm{~s}^{-1}\right)$, and $8.2^{\prime}(83 \mathrm{kpc})\left(2572 \mathrm{~km} \mathrm{~s}^{-1}\right)$.

SBS 1305+547 - A closely interacting pair consisting of an S0-type galaxy and a highly inclined disk galaxy centred $11 \operatorname{arcsec}(7 \mathrm{kpc})$ away. The S0-type galaxy could itself have two nuclei 3 arcsec ( $2 \mathrm{kpc}$ ) apart.

There are three much smaller galaxies $\sim 5.5^{\prime}(22 \mathrm{kpc})$ away from SBS $1305+547$ and at similar velocities.

A $3.0 \pm 0.5 \mathrm{mJy}$ unresolved radio counterpart lies at $130728.8+542655$ (J2000).

SBS 1317+523 A - This object has been classified as a merger, with four non-stellar components within a common envelope (Petrosian et al. 2002). The merger description is also based on its high absolute brightness ( -19.1 mag). It was observed (as a blue compact galaxy) in the HI $21 \mathrm{~cm}$ line by Thuan et al. (1999) who reported a total HI flux of $4.74 \pm 0.35 \mathrm{Jy} \mathrm{km} \mathrm{s}^{-1}$. The large difference in the HI flux from the present observation could be due to the very different beams of the Nancay and Effelsberg telescopes. Possible confusion with the S0a-type galaxy SBS $1723+523 \mathrm{~B}\left(V_{\text {hel }}=\right.$ $4684 \mathrm{~km} \mathrm{~s}^{-1} ; 41.5 \operatorname{arcsec}(13 \mathrm{kpc})$ south-east) and also from Mrk 0251 (4618 $\mathrm{km} \mathrm{s}^{-1}$ ) located $2.4 \operatorname{arcmin}(45 \mathrm{kpc})$ away.

A $5.5 \pm 1.1 \mathrm{mJy}$ radio counterpart of size $\sim 1^{\prime}$ along PA $125 \mathrm{deg}$ is centered at $131948.3+520401$ (J2000).

SBS $1435+516$ - The asymmetric narrow HI profile could have a contribution from the bright edge-on spiral centered 9.3' (92 kpc) away $\left(2212 \mathrm{~km} \mathrm{~s}^{-1}\right)$. A fainter galaxy at $8.7^{\prime}$ (86 kpc) $\mathrm{E}$ is also seen but its velocity is centered outside the observed profile and hence probably causes no confusion.

SBS 1436+529 - A merger system with two nuclei separated by $3.8 \operatorname{arcsec}(0.9 \mathrm{kpc})$. The brighter south-eastern nucleus is more centrally located.

A similarly bright galaxy SBS 1436+529B (3389 $\left.\mathrm{km} \mathrm{s}^{-1}\right)$ is located 7.8' (109 kpc) away. Hence, any contribution to the $\mathrm{HI}$ profile is expected to be strongly attenuated.

SBS 1443+499 - An Sa-type spiral galaxy in close interaction with a compact object located 7.4 arcsec to the north-east of the galaxy nucleus.

SBS 1444+557 - A marginally resolved radio counterpart with $12.6 \pm 0.9 \mathrm{mJy}$ lies at $144545.0+513450$ (J2000).

SBS $1452+540$ - According to Petrosian et al. (2002) and SDSS DR6 data (SDSS J145338.42+534759.1) this object resembles an S0-type galaxy with a starburst nucleus, which is in close interaction with another disky object. The two galaxies are connected with a tidal tail extending 14.6 arcsec $(3.3 \mathrm{kpc})$.

SBS 1504+514 - A merger system with two nuclei separated by $5.2 \operatorname{arcsec}(1.3 \mathrm{kpc})$. The south-eastern nucleus is more centrally located. HI observations of this galaxy have earlier been reported by Huchtmeier et al. (2005), with less RFI and a better baseline, compared to the present HI profile.

Any confusion from UGC $09702\left(3574 \mathrm{~km} \mathrm{~s}^{-1}\right)$ located $8^{\prime}$ southwest, although possible, must be severly attenuated due to the large offset from the beam centre.

A very faint $(\sim 1 \mathrm{mJy})$ radio counterpart lies at $150553.2+511508$ (J2000).

SBS 1509+583A - An extended radio counterpart of size $\sim 2$ arcmin at PA $\sim 125 \mathrm{deg}$ and a total flux of $13 \pm 2 \mathrm{mJy}$ lies at $151018.2+581057(\mathrm{~J} 2000)$.

SBS 1515+556 - Merger of a spiral galaxy with an elongated object which possibly itself has a two-component structure. The brighter of the two is $9.7 \operatorname{arcsec}(5.2 \mathrm{kpc})$ away from nucleus of the spiral galaxy.

SBS 1519+496 - A highly inclined bright spiral galaxy. A bright object is projected on its eastern edge, 9 arcsec from the nucleus of the spiral. This object has been observed as a "blue compact galaxy" by Thuan et al.(1999) who found an HI flux of $0.96 \pm 0.23 \mathrm{Jy} \mathrm{km} \mathrm{s}^{-1}$. The narrow (in RA) beam of the Nancay radio telescope might have tapered the HI emission.

SBS 1533+574AB - SBS 1533+574A and SBS 1533+574B are merging compact galaxies at a separation of 7.1 arcsec (1.6 kpc). This system has been designated as a merger also by Pustilnik et al. (2001). A marginally resolved radio counterpart with $6.0 \pm 1.5 \mathrm{mJy}$ lies at $153412.2+571656$ (J2000). 
SBS 1551+601AB - SBS1551+601 A and B are bright HII regions within a high surface brightness irregular galaxy of an absolute magnitude $\approx-15 \mathrm{mag}$. At least two more HII regions have been identified within this galaxy. All four HII regions form a chain-like structure inside the elongated body of this galaxy. The average separation between these HII regions is $2.7 \operatorname{arcsec}(0.5 \mathrm{kpc})$. This system has been described as a merger by Pustilnik et al. (2001).

SBS 1616+594A - A peculiar Sb-type spiral galaxy with a starburst nucleus forms a pair with the highly inclined spiral galaxy SBS 1616+594B at a separation of $54.4 \operatorname{arcsec}(16 \mathrm{kpc})$. This galaxy has been observed under the name UGC 10331 (PGC057731) by Theureau et al. (1998) who reported an HI flux of $4.3 \pm 1.2 \mathrm{Jy} \mathrm{kms}^{-1}$, in agreement with our measurement.

An unresolved radio counterpart of peak flux $\sim 7 \mathrm{mJy}$ lies at $161720.7+591920$ (J2000). It is strongly confused with a few times stronger, resolved radio source located $\sim 2^{\prime}$ (35 kpc) away at $\mathrm{PA} \sim 80 \mathrm{deg}$.

SBS 1657+590A - also known as NGC 6285 is a merger (Petrosian et al. 2002) with a LINER nucleus. It forms a physical pair with SBS $1657+590$ B. The position angle of a line connecting the nuclei of both galaxies is $\sim 40$ degrees. The relatively strong radio source $(149 \mathrm{mJy}$ at $1.4 \mathrm{GHz})$ is unresolved with the NVSS beam $\left(45^{\prime \prime}\right)$ but clearly resolved with the beam of the FIRST survey (Becker et al. 1985) and is extended along PA 40 degrees. Possibly this is a result of gravitational interaction between this galaxy pair. The upper limit in HI corresponds to $\sim 3 \times 10^{9} M_{\odot}$ (assuming a line width of $200 \mathrm{~km} \mathrm{~s}^{-1}$ ) which is not unusual for spiral galaxies.

SBS 1712+593AC - A closely interacting pair (Arp32 = VV89 = KPG506) consisting of an irregular and a SBm-type galaxy separated by $40 \operatorname{arcsec}(3 \mathrm{kpc})$.

\section{Appendix B: Description of Table 2}

The derived global parameters for the objects for which we found no evidence of a substantial confusion of the HI profile are presented in Table 2: the galaxy name in Col. 1, the optical heliocentric velocity (Table 1, Col. 5) has been reduced to the frame of the cosmic microwave background (using NED), $V_{3 \mathrm{Kbgd}}(\mathrm{Col}$. 2). We did not use the more accurate HI velocities because of the possibility of confusion in some cases. The distances in column 3 have been derived taking a Hubble constant $H_{0}=72 \mathrm{~km} \mathrm{~s}^{-1} \mathrm{Mpc}^{-1}$ (Freedman et al. 2001). Optical diameters in the $\mathrm{D}_{25}$ system (Table 1, Col. 3) have been corrected for absorption and for the inclination, derived from the axial ratio, assuming an intrinsic axial ratio of 0.2 (e.g. Tully 1985, in view of the uncertainties in these inclinations we did not apply corrections for possible dependance of the intrinsic axial ratio on the galaxy type):

$\log a_{0}=\log a+0.09 A_{\mathrm{b}}-0.2 \log (a / b)$,

where the foreground absorption in the blue, $A_{\mathrm{b}}$, is from Schlegel et al. (1998), as given in the NED.

The computed linear diameter $A_{0, i}[\mathrm{kpc}]$ follows in Col. 4 and the absolute magnitude $M_{\mathrm{b}, t}^{0, i}$ corrected for Galactic extinction (Schlegel et al. 1998) and internal absorption in a galaxy, $A_{i}$, on its inclination and luminosity is (Giovanelli et al. 1994; Tully et al. 1998; Verheijen 2001; Karachentsev et al. 1999):

$A_{i}=\left[1.6+2.8\left(\log V_{m}-2.2\right)\right] \times \log (a / b) ; \quad V_{m} \geq 42.7 \mathrm{~km} \mathrm{~s}^{-1}$

$A_{i}=0-\quad$ other

$A_{i}=0-\mathrm{E}, \mathrm{S} 0, \mathrm{dSph}$.

Here we use the following definition of $V_{m}$ :

The HI rotational velocity $V_{m}=W_{50} /(2 \sin i)$ corrected for inclination and turbulent motion (Tully \& Fouqué 1985) with isotropic non-circular motion parameter $\sigma_{z}=8 \mathrm{~km} \mathrm{~s}^{-1}$.

The total H I mass (Col. 6) was calculated using

$M_{\mathrm{HI}}=2.355 \times 10^{5} D^{2} \int S_{v} \mathrm{~d} v$,

where $D$ is the distance in Mpc and $\int S_{v} \mathrm{~d} v$ is the integrated HIflux in $\mathrm{Jy} \mathrm{km} \mathrm{s}^{-1}$ seen within the 9.3' antenna beam (including confusing sources).

Data in the subsequent columns are provided only for single galaxies (mergers), i.e., systems having no known source of confusion within the antenna beam.

The total mass $M_{\mathrm{T}}(\mathrm{Col} .7)$ has been derived from:

$M_{\mathrm{T}}=$ const. $D a_{0} \Delta v_{0, i}^{2}$,

where $D$ is the distance in Mpc, $a_{0}$ the corrected optical diameter in arcmin, const. $=33110$, and $\Delta v_{0, i}$ the corrected edge-on linewidth at $50 \%$ of the peak flux (e.g. Karachentsev et al. 2004).

The HI mass-to-luminosity ratio $M_{\mathrm{HI}} / L_{B}$, the mass-toluminosity ratio $M_{\mathrm{T}} / L_{B}$, and the relative HI mass $M_{\mathrm{HI}} / M_{\mathrm{T}}$ follow in Cols. 8 to 10 , respectively.

The radio continuum parameters of the detected BCDGs are based on the 1.4 GHz survey NVSS (Condon et al. 1998). 